\title{
General Perturbation Method for Satellite Constellation Reconfiguration Using Low-Thrust Maneuvers
}

\author{
Ciara N. McGrath* and Malcolm Macdonald \\ University of Strathclyde, Glasgow, Scotland G1 1XJ, United Kingdom
}

DOI: $10.2514 / 1 . G 003739$

\begin{abstract}
A general perturbation solution to a restricted low-thrust Lambert rendezvous problem, considering circular-tocircular in-plane maneuvers using tangential thrust and including a coast arc, is developed. This provides a fully analytical solution to the satellite reconnaissance problem. The solution requires no iteration. Its speed and simplicity allow problems involving numerous spacecraft and maneuvers to be studied; this is demonstrated through two case studies. In the first, a range of maneuvers providing a rapid flyover of Los Angeles is generated, giving an insight to the trade space and allowing the maneuver that best fulfills the mission to be selected. A reduction in flyover time from 13.8 to 1.6 days is possible using a less than $17 \mathrm{~m} / \mathrm{s}$ velocity change. A comparison with a numerical propagator including atmospheric friction and an 18th-order tesseral model shows $4 \mathrm{~s}$ of difference in the time of flyover. A second study considers a constellation of 24 satellites that can maneuver into repeating ground track orbits to provide persistent coverage of a region. A set of maneuvers for all satellites is generated for four sequential targets, allowing the most suitable maneuver strategy to be selected. Improvements in coverage of greater than $\mathbf{1 0}$ times are possible as compared to a static constellation using $35 \%$ of the propellant available across the constellation.
\end{abstract}

\section{Nomenclature}

$A=$ propulsive acceleration, $\mathrm{m} / \mathrm{s}^{2}$

$A_{\text {atm }}=$ acceleration due to atmospheric friction, $\mathrm{m} / \mathrm{s}^{2}$

$a \quad=$

$\bar{a}=$

$C_{D}=$

d $=$

e $=$

$f=$

$H=$

h $=$

$i \quad=$

$J_{2}=$

$k=$

$M=$

$m=$

$n=$

$\bar{n} \quad=$

$R_{e}=$

$s=$

$t=$

$U \quad=$

$u=$

$v=$

$X \quad=$

$\Delta u=$

$\Delta V=$

osculating semimajor axis, $\mathrm{km}$

mean semimajor axis, $\mathrm{km}$

satellite coefficient of drag eccentricity

flattening of Earth

scale height of Earth's atmosphere, $\mathrm{km}$

altitude with respect to mean Earth radius, $\mathrm{km}$

inclination, deg

the second degree

utility function weighting factor

mean anomaly, deg

satellite mass, $\mathrm{kg}$

mean motion, deg

perturbed mean motion, deg

mean Earth radius, $\mathrm{km}$

instrument swath width, $\mathrm{km}$

time, $\mathrm{s}$

utility function

satellite velocity, $\mathrm{m} / \mathrm{s}$

attribute of utility function

change in argument of latitude, deg

change in velocity, $\mathrm{m} / \mathrm{s}$

effective cross-sectional area, $\mathrm{m}^{2}$

$=$ latitude, deg

$=$ true anomaly, deg

$=$ atmospheric density, $\mathrm{kg} / \mathrm{m}^{3}$ great-circle distance from subsatellite point to target, $\mathrm{km}$

coefficient of the Earth's gravitational zonal harmonic of

argument of latitude, deg

change in right ascension of the ascending node, deg

$=$ standard gravitational parameter of Earth, $\mathrm{m}^{3} / \mathrm{s}^{2}$

Received 10 April 2018; revision received 2 October 2018; accepted for publication 9 April 2019; published online Open Access 27 June 2019. Copyright $@ 2019$ by Ciara N. McGrath. Published by the American Institute of Aeronautics and Astronautics, Inc., with permission. All requests for copying and permission to reprint should be submitted to CCC at www. copyright.com; employ the eISSN 1533-3884 to initiate your request. See also AIAA Rights and Permissions www.aiaa.org/randp

*Research Associate, Department of Mechanical and Aerospace Engineering, 75 Montrose Street. Professional Member AIAA.

Reader, Department of Mechanical and Aerospace Engineering, 75 Montrose Street. Associate Fellow AIAA.

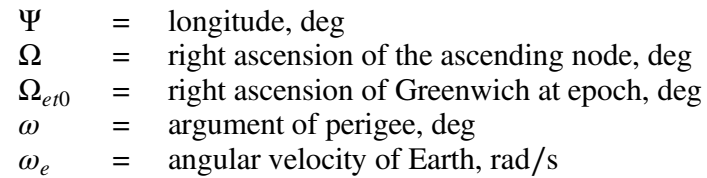

Subscripts

alt $=$ total for altitude changing phases (that is, phase 1 and phase 3)

$c=$ geocentric

POI $=$ point of interest

ref $=$ reference value

$\mathrm{SSP}=$ subsatellite point

total $=$ total at maneuver end

$0=$ at maneuver start

$1=$ at end of phase 1

$2=$ at end of phase 2

$3=$ at end of phase 3

\section{Introduction}

T RADITIONALLY, low-Earth-orbiting (LEO) Earth observation constellations have aimed to provide global coverage [1-7]. This focus on spatial performance can make providing good temporal coverage challenging and responding to a change in mission requirements difficult [ $[-10]$. This paper looks to enable responsive, reconfigurable LEO satellite constellations by providing a rapid solution and holistic insight to the satellite reconnaissance problem. Note that reconfiguration here, and throughout this paper, is taken to mean the changing of a constellation from one formation to another through the maneuvering of the constituent satellites. The solution developed uses a general perturbation method to calculate circularto-circular in-plane low-thrust satellite maneuvers that enable a point of interest on the Earth's surface to be targeted. This provides a fully analytical solution to the low-thrust Lambert rendezvous problem with tangential thrust and a single coast arc. It is of note that the solution is therefore also directly applicable for other purposes, such as maneuver design for in-orbit rendezvous or close approach: for example, in support of in-orbit servicing or space debris removal applications. However, in this paper, the solution is further extended to the spacecraft's ground track. Most recent attempts to solve the Lambert targeting problem have focused on increasing the efficiency of the available numerical methods [11-13]. However, a fully analytical solution is extremely fast and noniterative, making it ideal for preliminary trajectory design. The analytical solution presented in 
Ref. [14] demonstrates this for a single-impulse transfer; the solution presented herein for a low-thrust spiral transfer with a single coast arc shares many of these advantages.

Providing overflight of multiple successive ground targets, also known as satellite reconnaissance, using a satellite equipped with electric propulsion was apparently first proposed by Guelman and Kogan in 1999 [15]. They determined the problem to be a discrete optimization problem and used a simulated annealing method to identify the minimum propellant trajectory that would satisfy the requirement to pass over a set of targets in a given time. Jean and de Lafontaine extended this work by incorporating the first harmonic of the Earth's oblateness and generalizing the method to give an expression for the time to target flyby following in-plane low-thrust maneuvers described by cubic or quartic guidance laws [16]. They then incrementally searched through the possible solutions to find a trajectory with an acceptable acceleration profile.

The use of high-thrust maneuvers to provide overflight of a target has also been studied. Zhu et al. proposed a solution based on a Hohmann transfer to achieve flyover of a given ground target [17]. Multiple objectives are considered, including maximizing coverage time of the target and minimizing the propellant required for the orbit transfer. Combining these into a fitness function, Zhu et al. used particle swarm optimization and differential evolution to select a satellite from an available set and to optimize the maneuver trajectory. However, Zhu et al. noted that an optimal solution cannot be guaranteed. This is a weakness of all numerical techniques that have been used to tackle this problem to date, for both low- and high-thrust scenarios, because they do not provide a complete view of the solution space.

Work has also been done considering the reconfiguration of a constellation of multiple satellites. Davis proposed the use of Hohmann transfer maneuvers and approximated the solution to Lambert's problem using a fourth-order polynomial [18]. He then used an auction algorithm, as proposed by De Weck et al. [19], to assign the satellites to appropriate slots in the final constellation. Consideration has also been given to the design of a constellation to enable efficient future reconfiguration. Legge highlighted that traditional constellation designs are nonoptimal for reconfigurable missions and that, when designing for reconfiguration, unusual constellation architectures can offer improved performance [20]. Legge's work used an analytical method to describe the constellation reconfiguration using impulsive double Hohmann transfers. He then performed a multiobjective optimization to assign satellites to positions in the new constellation and identify favorable configurations. Legge suggested that consideration of low-thrust electric propulsion would be complementary to his work because it could allow for lower propellant usage at the cost of increased reconfiguration time. A tool developed by Paek et al. in recent years aimed to concurrently optimize satellite and constellation designs by running numerous reconfigurations and using a genetic algorithm [21]. Paek et al. limited the maximum constellation size to 49 satellites to reduce computation time, but they still required up to $15 \mathrm{~h}$ to perform a single optimization. Based on these results, Paek et al. highlighted that the length of time required to propagate the satellite maneuvers is a key limitation of his tool.

There have been some recent attempts to solve the reconnaissance problem analytically. Zhang et al.'s [22] and Zhang and Sheng's [23] work provided approximate semianalytical solutions to the problem using high-thrust propulsion. Their methods, based on Kepler's equations and considering up to four impulsive maneuvers, allow for the minimum-energy trajectory to be found. The results are found to be suboptimal as compared with a numerical solver, but Zhang et al. [22] and Zhang and Sheng [23] highlighted that they could provide an initial guess for higher-precision models and may be useful in cases where fast computational speed is desired. For low-thrust propulsion, Co et al. $[24,25]$ and Co and Black [26] developed a control algorithm that allows a single satellite to pass over a given target using electric propulsion. Co et al. $[24,25]$ and Co and Black [26] used the difference in time of flyover between the maneuvering satellite and the nonmaneuvering reference satellite as a metric, and they could solve for this using a single equation. Their method numerically propagates the reference satellite's position for a given time in order to identify close passes to the target. These passes are then ordered in terms of the soonest encounter if the fastest overflight solution is desired, or by order of closest pass if the minimum propellant solution is desired. Discarding those that are infeasible allows the fastest, or minimumenergy, feasible solution to be found. Being based on a straightforward analytical expression, Co et al.'s [24,25] and Co and Black's [26] solution allows for analysis of the possible reach of a maneuver of this type and the impact of the initial orbit parameters on the maneuver efficiency. However, its accuracy is limited because it does not consider the perturbing effect of an oblate central body on the satellite mean motion; this simplification introduces significant errors when considering the position of the satellite's ground track. The requirement to numerically propagate the position of the reference satellite is also a limitation of the solution because the length of time required for the procedure can limit its applicability for large-scale constellations or complex scenarios involving multiple targets.

The general perturbation method presented herein offers a fast, analytical solution to the satellite reconnaissance problem. A full overview of the solution space is produced for each scenario, enabling the mission designer or operator to obtain an insight into the reconfiguration problem and select the maneuvers best suited to the mission objective. The method is restricted to circular-to-circular inplane tangential low-thrust maneuvers and incorporates a coast arc to ensure efficient propellant usage. The solution directly links the satellite maneuvers to the resulting ground track location, allowing for straightforward overflight targeting of a given region. Two disaster response case studies are presented to demonstrate the ease with which complex scenarios can be investigated to gain vital insights that would be difficult to obtain through numerical methods alone.

\section{Method}

To develop an analytical solution to the overflight targeting problem, the Gauss-Lagrange planetary equations are used and only secular perturbations of the orbit are considered. In the case of the Earth, one of the most significant secular perturbations is caused by the first zonal harmonic of the nonspherical gravitational field (also known as $J_{2}$ ). This produces a secular variation in the right ascension of the ascending node (RAAN) of the satellite $\Omega$; the argument of perigee $\omega$; the mean anomaly $M$; and the mean motion $n[27,28]$. The argument of latitude (AOL) $u$, defined as the sum of the argument of perigee and the true anomaly $\theta$ (i.e., $u=\omega+\theta$ ) also experiences a secular change as a result of $J_{2}$. The rate of change of each of these orbital elements due to $J_{2}$ is a function of the satellite's semimajor axis. Changing a satellite's altitude has a direct effect on the orbit period and can be used to affect a change in the satellite's true anomaly, and thus the satellite ground track. Including the secular effects of $J_{2}$ gives a more accurate prediction of this change by incorporating the perturbing effects of the central body on the satellite mean motion, the AOL, and the RAAN. This accuracy is necessary when targeting the overflight of localized regions of the Earth's surface because a small difference in the RAAN or AOL can give large errors in the predicted location of the satellite ground track.

Other disturbing forces, such as higher-order central body effects, third-body effects, solar radiation pressure, and atmospheric friction, can also produce secular effects in the orbital elements. As will be discussed in Sec. III, the inaccuracies in the solution arising from excluding higher-order central body effects were found to be small, and thus it was deemed acceptable to exclude them from the solution. Third-body effects for LEO satellites are very small when compared with the effect of $J_{2}$; as such, they are not considered in the solution. Additionally, the effects of atmospheric friction at LEO altitudes are significantly larger than the effects of solar radiation, and so solarradiation effects are not considered. Atmospheric friction can have a notable impact on the satellite orbit, and its consideration for longduration scenarios is vital. Assuming that atmospheric friction compensation maneuvers are performed throughout the coast arc allows the assumption of no altitude change during this time. However, in order to maintain the analytical solution, the effect of 
atmospheric friction is not considered during the altitude change phases. Due the relatively short duration of these phases (less than four days for all cases considered), the errors introduced by this simplification are small, as will be shown in Sec. III.

\section{A. Maneuver Description}

The satellite maneuver strategy considered in this paper is a threephase maneuver that consists of an initial thrusting phase in which the satellite increases or decreases its altitude relative to its initial orbit: hereafter referred to as phase 1 . This is done using continuous low thrust with a constant acceleration. The second phase, referred to as phase 2 , is a coast arc in which the satellite is assumed to maintain a constant altitude by thrusting to counteract the effect of atmospheric friction. In the final phase, denoted phase 3 , the satellite moves to the desired final altitude; this may or may not be the same as the initial altitude at the beginning of phase 1 . This is performed using continuous low-thrust propulsion with the same constant acceleration as in phase 1 . This method is similar to the three-sequence transfer found by Cerf to offer the minimum propellant low-thrust transfer between circular orbits when the effects of $J_{2}$ are included [29]. Note that not all phases are required to be present for the solution to be valid; one or more of the phases can be excluded without fundamentally altering the method.

To solve the overflight targeting problem, an analytical expression is created that defines the change in the RAAN and the AOL achieved during this three-phase maneuver as a function of the total time taken and the change in velocity $\Delta V$ required for the maneuver. This change in the RAAN and AOL is then linked to the spacecraft ground track to determine the location of the subsatellite point after the three-phase maneuver has been performed. Selecting the final altitude to be that of a repeating ground track orbit can additionally provide regular, repeated coverage of the target region, if desired.

\section{B. Fundamental Equations}

The time rate of change of a satellite's orbital elements can be expressed as a sum of the conservative and nonconservative perturbing forces acting on the satellite [30]. The well-known Lagrange planetary equations, presented in Refs. [31] and [32], provide a convenient method to determine the effect of conservative forces acting on the satellite; whereas the Gauss version of the planetary equations, presented in Ref. [32], is more appropriate for the consideration of nonconservative perturbations, such as those caused by a constant applied acceleration.

The Lagrange planetary equations describe the time rate of change of the orbital elements as a result of a disturbing function. To consider the secular effects of $J_{2}$ only, the relevant disturbing function can be averaged over one orbit period. This procedure was described in Refs. [31] and [32]. For a circular orbit, the Lagrange planetary equations, considering only the secular effects of $J_{2}$, reduce to

$$
\begin{gathered}
\left(\frac{\mathrm{d} \bar{a}}{\mathrm{~d} t}\right)_{J_{2}}=0 \\
\left(\frac{\mathrm{d} e}{\mathrm{~d} t}\right)_{J_{2}}=0 \\
\left(\frac{\mathrm{d} i}{\mathrm{~d} t}\right)_{J_{2}}=0 \\
\left(\frac{\mathrm{d} \Omega)_{J_{2}}}{\mathrm{~d} t}=-\frac{3 \bar{n} R_{e}^{2} J_{2}}{2 \bar{a}^{2}} \cos i\right. \\
\left(\frac{\mathrm{d} \omega}{\mathrm{d} t}\right)_{J_{2}}=\frac{3 \bar{n} R_{e}^{2} J_{2}}{4 \bar{a}^{2}}\left(4-5 \sin ^{2} i\right)
\end{gathered}
$$

$$
\left(\frac{\mathrm{d} M}{\mathrm{~d} t}\right)_{J_{2}}=\bar{n}=n\left[1-\frac{3 R_{e}^{2} J_{2}}{4 \bar{a}^{2}}\left(3 \sin ^{2} i-2\right)\right]
$$

where $\bar{n}$ is the mean motion including secular $J_{2}$ perturbations. The unperturbed mean motion $n$ is defined as $n=\sqrt{\mu / \bar{a}^{3}}$.

The Gaussian form of the Lagrange planetary equations provides a description of the time rate of change of the orbital elements as a result of perturbations that are expressed as disturbing accelerations or specific forces. The standard form of the Gauss planetary equations are given by Refs. [32] and [33]. The only nonconservative force directly considered is the spacecraft propulsion system acceleration; atmospheric friction does produce a nonconservative force, but it is assumed to be compensated for during any coast arcs and is neglected during the altitude change phases in order to maintain the analytical solution. The spacecraft acceleration $A$ will be continually applied in the tangential direction, with a positive $A$ corresponding to a propulsive acceleration applied in the positive tangential direction. As this acceleration is assumed to be small for low-thrust systems, the orbit can be assumed to remain circular throughout the maneuver such that there will be no change in eccentricity. In addition, as the propulsion acceleration is assumed to be constant throughout the altitude change phases, all periodic terms (i.e., those that are a function of the true anomaly) can be ignored because the resulting perturbations will be zero when averaged over a single orbit period. The resulting equations of motion are thus

$$
\begin{aligned}
& \left(\frac{\mathrm{d} \bar{a}}{\mathrm{~d} t}\right)_{\text {thrust }}=\frac{2}{\bar{n}} A \\
& \left(\frac{\mathrm{d} e}{\mathrm{~d} t}\right)_{\text {thrust }}=0 \\
& \left(\frac{\mathrm{d} i}{\mathrm{~d} t}\right)_{\text {thrust }}=0 \\
& \left(\frac{\mathrm{d} \Omega}{\mathrm{d} t}\right)_{\text {thrust }}=0 \\
& \left(\frac{\mathrm{d} \omega}{\mathrm{d} t}\right)_{\text {thrust }}=0 \\
& \left(\frac{\mathrm{d} M_{0}}{\mathrm{~d} t}\right)_{\text {thrust }}=0
\end{aligned}
$$

Summing Eqs (1-12) gives full expressions for the time rate of change of the orbital elements under the influence of secular $J_{2}$ effects and with constant applied propulsive acceleration. These are

$$
\frac{\mathrm{d} \bar{a}}{\mathrm{~d} t}=\left(\frac{\mathrm{d} \bar{a}}{\mathrm{~d} t}\right)_{J_{2}}+\left(\frac{\mathrm{d} \bar{a}}{\mathrm{~d} t}\right)_{\text {thrust }}=\frac{2}{\bar{n}} A
$$

$$
\frac{\mathrm{d} e}{\mathrm{~d} t}=\left(\frac{\mathrm{d} e}{\mathrm{~d} t}\right)_{J_{2}}+\left(\frac{\mathrm{d} e}{\mathrm{~d} t}\right)_{\text {thrust }}=0
$$$$
\frac{\mathrm{d} i}{\mathrm{~d} t}=\left(\frac{\mathrm{d} i}{\mathrm{~d} t}\right)_{J_{2}}+\left(\frac{\mathrm{d} i}{\mathrm{~d} t}\right)_{\text {thrust }}=0
$$

$$
\frac{\mathrm{d} \Omega}{\mathrm{d} t}=\left(\frac{\mathrm{d} \Omega}{\mathrm{d} t}\right)_{J_{2}}+\left(\frac{\mathrm{d} \Omega}{\mathrm{d} t}\right)_{\text {thrust }}=-\frac{3 \bar{n} R_{e}^{2} J_{2}}{2 \bar{a}^{2}} \cos i
$$




$$
\begin{gathered}
\frac{\mathrm{d} \omega}{\mathrm{d} t}=\left(\frac{\mathrm{d} \omega}{\mathrm{d} t}\right)_{J_{2}}+\left(\frac{\mathrm{d} \omega}{\mathrm{d} t}\right)_{\text {thrust }}=\frac{3 \bar{n} R_{e}^{2} J_{2}}{4 \bar{a}^{2}}\left(4-5 \sin ^{2} i\right) \\
\frac{\mathrm{d} M}{\mathrm{~d} t}=\left(\frac{\mathrm{d} M}{\mathrm{~d} t}\right)_{J_{2}}+\left(\frac{\mathrm{d} M}{\mathrm{~d} t}\right)_{\text {thrust }}=n\left[1-\frac{3 R_{e}^{2} J_{2}}{4 \bar{a}^{2}}\left(3 \sin ^{2} i-2\right)\right]=\bar{n}
\end{gathered}
$$

As the argument of perigee is undefined for circular orbits, it is helpful to consider the change in the argument of latitude instead. For a circular orbit, $\theta=M$; and the rate of change of the argument of latitude can be defined as

$$
\frac{\mathrm{d} u}{\mathrm{~d} t}=\frac{\mathrm{d} M}{\mathrm{~d} t}+\frac{\mathrm{d} \omega}{\mathrm{d} t}=\bar{n}+\frac{3 \bar{n} R_{e}^{2} J_{2}}{4 \bar{a}^{2}}\left(4-5 \sin ^{2} i\right)
$$

\section{Conversion to Mean Orbital Elements}

Equations (13-18), described in Sec. II.B, include thrust and secular perturbations resulting from the first zonal harmonic $J_{2}$; no periodic contributions are considered due to the averaging of the disturbing function [31]. The resulting orbital elements calculated using these expressions are thus essentially mean elements because both the short-periodic contributions (related to the satellite orbit period) and the long-periodic contributions (related to the motion of the line of apsides) have been ignored. Hence, for a consistent solution, mean elements should be used in the calculation of these expressions. There are many accepted methods to calculate mean orbital elements; for the analysis presented herein, Brouwer's definition of mean orbital elements is used [28]. The use of a different mean element set will impact the results obtained; however, this does not affect the fundamental method presented in this paper.

Of particular note is the conversion from an osculating to a mean semimajor axis. This was given by Brouwer [28] as

$$
\bar{a}=a-\frac{3 J_{2} R_{e}^{2}}{2 a} \sin ^{2}(i) \cos (2 u)
$$

As will be discussed in Sec. II.D, forming a fully analytical solution to describe the three-phase transfer requires that the thrusting phases be integrated over the semimajor axis rather than over time. If the osculating semimajor axis is to be given as an input, then it is necessary to incorporate the conversion from osculating to mean semimajor axis directly into the expressions presented in Sec. II.B. This allows the expressions to be integrated over the osculating, rather than the mean, semimajor axis and maintain the greatest accuracy. However, including the conversion to the mean semimajor axis in Eq. (13) prevents a fully analytical solution from being obtained due to the complex functions produced during integration. To avoid this issue, the osculating semimajor axis can be used in Eq. (13) with minimal impact on the solution accuracy, as will be shown in Sec. III. All other orbital elements are taken as their value at the time the osculating value is given; for the cases presented herein, all osculating values are given at the time of the most recent pass of the ascending node (i.e., $u=0 \mathrm{deg}$ ). To directly compare with numerical solutions, this is the method used throughout this paper, allowing the osculating elements to be used as inputs to both the numerical and analytical methods. This gives the rate of change of the semimajor axis as

$$
\frac{\mathrm{d} a}{\mathrm{~d} t}=\frac{2}{\bar{n}^{\prime}} A
$$

where

$$
\bar{n}^{\prime}=n\left[1-\frac{3 R_{e}^{2} J_{2}}{4 a^{2}}\left(3 \sin ^{2} i-2\right)\right]
$$

\section{Change in Right Ascension of Ascending Node and Argument of Latitude}

The rate of change of the RAAN and AOL are described by Eqs. (16) and (19), respectively. During the coast arc, because atmospheric friction compensation maneuvers are assumed to be performed, the semimajor axis $a$ can be assumed to remain constant and Eqs. (16) and (19) can be integrated over time to produce expressions for the change in the RAAN and AOL.

During the altitude change phases, the semimajor axis is not constant and varies according to Eq. (21). As a result, Eqs. (16) and (19) cannot be directly integrated over time. By combining Eqs. (21) and (16), an expression for the change in the RAAN as a function of the semimajor axis can be produced in the form of

$$
\frac{\mathrm{d} \Omega}{\mathrm{d} a}=-\frac{3 \bar{n} \bar{n}^{\prime} R_{e}^{2} J_{2}}{4 \bar{a}^{2} A} \cos i
$$

Assuming that acceleration remains constant throughout the altitude change maneuver, Eq. (23) can be integrated with respect to the semimajor axis to give the change in the RAAN over the course of the altitude change maneuver. Similarly, combining Eqs. (21) and (19) gives an expression for the change in the AOL as a function of the semimajor axis as

$$
\frac{\mathrm{d} u}{\mathrm{~d} a}=\frac{\bar{n} \bar{n}^{\prime}}{2 A}\left(1+\frac{3 R_{e}^{2} J_{2}}{4 \bar{a}^{2}}\left(4-5 \sin ^{2} i\right)\right)
$$

This can be integrated with respect to the semimajor axis to give the change in the AOL over the course of the altitude change maneuver.

\section{E. Analytical Description of Three-Phase Maneuver}

As described in Sec. II.D, integrating Eqs. (16) and (19) gives expressions for the change in the RAAN and the change in the AOL, respectively, for the satellite during the coast arc as a function of time. This corresponds to phase 2 of the three-phase maneuver. Integrating Eqs. (23) and (24) gives expressions for the change in the RAAN and AOL, respectively, as a function of the change in the semimajor axis for a satellite performing an altitude change maneuver. This corresponds to phases 1 and 3 of the three-phase maneuver. Combining these equations to account for all three phases gives fully analytical expressions for the RAAN and the AOL of the satellite after the maneuver is complete. That is,

$$
\Omega_{\mathrm{total}}=\Omega_{0}+\Delta \Omega_{1}+\Delta \Omega_{2}+\Delta \Omega_{3}
$$

and

$$
u_{\text {total }}=u_{0}+\Delta u_{1}+\Delta u_{2}+\Delta u_{3}
$$

These expressions, although complete, express the final RAAN and AOL in terms of the semimajor axis reached at the end of phase 1 $a_{1}$ and the time required for the coasting phase $t_{2}$. For the purposes of this work, it is more useful to express these changes in terms of the total maneuver time $t_{\text {total }}$ and the required change in velocity. The time required for the coast arc $t_{2}$ can be expressed as

$$
t_{2}=t_{\text {total }}-t_{1}-t_{3}
$$

where $t_{1}$ and $t_{3}$ are the time taken for phase 1 and phase 3, respectively. Integrating Eq. (21) with respect to time and rearranging gives an expression for $t_{1}$ as

$$
t_{1}=\frac{\sqrt{\mu}\left(a_{0}^{5 / 2}\left\{20 a_{1}^{2}+3 J_{2} R_{e}^{2}\left[2-3 \sin ^{2} i\right]\right\}+3 a_{1}^{5 / 2} J_{2} R_{e}^{2}\left[3 \sin ^{2} i-2\right]-20 a_{1}^{5 / 2} a_{0}^{2}\right)}{20 a_{0}^{5 / 2} a_{1}^{5 / 2} A}
$$


with $t_{3}$ being calculated using the same expression but with $a_{2}$ in place of $a_{0}$ and $a_{3}$ in place of $a_{1}$. Substituting these into Eq. (27), with the assumption that $a_{2}=a_{1}$, and then into Eqs. (25) and (26) allows $\Omega_{\text {total }}$ and $u_{\text {total }}$ to be expressed in terms of $t_{\text {total }}$ and $a_{1}$.

It is then possible to express $a_{1}$ in terms of the change in velocity required to change the altitude of the satellite $\Delta V_{\text {alt }}$. Assuming a small propellant mass flow rate and a small propellant mass fraction [34], the change in velocity required to change the satellite altitude in phase 1 can be approximated as

$$
\Delta V_{1}=\left|\sqrt{\frac{\mu}{a_{1}}}-\sqrt{\frac{\mu}{a_{0}}}\right|
$$

with $\Delta V_{3}$ calculated using a similar expression but with $a_{3}$ in place of $a_{0}$. From this, an expression for $a_{1}$ as a function of $\Delta V_{\text {alt }}$ can be derived as

$$
a_{1}=\frac{4 \mu a_{0}}{\mu+a_{0}\left[\sqrt{\left(\mu / a_{3}\right)} \pm \Delta V_{\text {alt }}\right]\left[2 \sqrt{\left(\mu / a_{0}\right)}+\sqrt{\left(\mu / a_{3}\right)} \pm \Delta V_{\text {alt }}\right]}
$$

where $\Delta V_{\text {alt }}=\Delta V_{1}+\Delta V_{3}$. Note that $\Delta V_{\text {alt }}$ does not include the change in velocity required for atmospheric friction compensation $\Delta V_{2}$; this is discussed in more detail in Sec. II.G. In Eq. (30), $\Delta V_{\text {alt }}$ is additive for an altitude-lowering maneuver in which the satellite decreases its altitude in phase 1 and increases it in phase 3 , whereas $\Delta V_{\text {alt }}$ is subtractive for an altitude-raising maneuver in which the satellite raises its altitude in phase 1 and lowers it in phase 3 . Using this definition of $a_{1}$ in Eqs. (25) and (26), and assuming that the initial and final altitudes of the satellite are known, fully analytical expressions are produced for the RAAN and AOL at the end of the maneuver as a function of the total time required for the maneuver and the change in velocity required to change the satellite altitude. These expressions are given in full in the Appendix. Note that these equations are combined in such a way that only a single propulsive acceleration value for $A$ is required; in the case that the satellite raises its altitude in phase 1, a positive value of $A$ should be used; whereas if the satellite lowers its altitude in phase 1, a negative value of $A$ should be used.

\section{F. Analytical Description of Ground Track Motion}

If the aim of a given mission is to target a specific point of interest (POI) on the ground, then it is useful to link the RAAN and the AOL of the satellite postmaneuver to changes in the ground track. Using spherical geometry, the geocentric latitude of the subsatellite point (SSP) $\delta_{c}$ at a given time can be calculated by

$$
\delta_{c}=\sin ^{-1}\left(\sin i \sin u_{\mathrm{total}}\right)
$$

with the corresponding longitude of the SSP $\Psi_{\mathrm{SSP}}$ given as

$$
\Psi_{\mathrm{SSP}}=\operatorname{atan} 2\left(\frac{\cos i \sin u_{\text {total }}}{\cos u_{\text {total }}}\right)-\omega_{e} t_{\text {total }}+\Omega_{\text {total }}-\Omega_{e t 0}
$$

where $\Omega_{e t 0}$ is the right ascension of Greenwich at epoch, and $\omega_{e}$ is the angular rate of rotation of the Earth [35]. The geocentric latitude $\delta_{c}$ can be converted to geodetic latitude $\overline{\delta_{\mathrm{SSP}}}$ using

$$
\tan \delta_{\mathrm{SSP}}=\frac{\tan \delta_{c}}{1-f(2-f)}
$$

where $f$ is the flattening of the Earth [36]. Assuming a World Geodetic System 1984 ellipsoid model of the Earth [37], $f=0.00335281$.
The haversine formula given by

$$
\begin{aligned}
d & =2 R_{e} \sin ^{-1} \\
& \times\left[\sqrt{\sin ^{2}\left(\frac{\delta_{\mathrm{SSP}}-\delta_{\mathrm{POI}}}{2}\right)+\cos \left(\delta_{\mathrm{SSP}}\right) \cos \left(\delta_{\mathrm{POI}}\right) \sin ^{2}\left(\frac{\Psi_{\mathrm{SSP}}-\Psi_{\mathrm{POI}}}{2}\right)}\right]
\end{aligned}
$$

can be used to calculate the great-circle distance between the subsatellite point and the point of interest on the ground after the maneuver, assuming the latitude $\delta_{\mathrm{POI}}$ and longitude $\Psi_{\mathrm{POI}}$ of the point of interest are known [38]. Geodetic latitude values should be used in Eq. (34) for both the SSP and POI, although it should be noted that use of the haversine formula assumes a spherical Earth for the calculation of the distance between the points. All reference to "distance" throughout this paper refers to the great-circle distance calculated in this manner. Using Eqs. (31) and (32), the haversine distance $d$ can be expressed analytically in terms of the orbit elements, and thus using Eqs. (25) and (26) as a function of the maneuver time $t_{\text {total }}$ and $\Delta V_{\text {alt }}$. The partial derivative of Eq. (34) with respect to $t_{\text {total }}$ can be used to find the extrema of this distance function for a given $\Delta V_{\text {alt }}$, whereas the second partial derivative can be used to discern the minima from the maxima. Both the first and second derivatives of $d$ can be found analytically. This allows all minimum distance solutions to be found and those for which the point of interest is in view to be identified. The analytical nature of the solution means it can be solved quickly to provide a full overview of the solution space.

\section{G. Atmospheric Friction Compensation in Phase 2}

It is assumed that the satellite maintains a constant altitude during phase 2 (the coast arc) by thrusting in the positive tangential direction to compensate for the effect of atmospheric friction. The acceleration caused by this force can be approximated by

$$
\boldsymbol{A}_{\mathrm{atm}}=-\frac{1}{2} \frac{C_{D} \alpha}{m} \rho v_{\mathrm{rel}}^{2} \frac{\boldsymbol{v}_{\mathrm{rel}}}{\left|\boldsymbol{v}_{\mathrm{rel}}\right|}
$$

where $v_{\text {rel }}$ is the relative velocity between the satellite and the atmosphere [39]. Making the assumption that the atmosphere is static and that the atmospheric friction force acts only in the negative tangential direction gives

$$
A_{\mathrm{atm}}=-\frac{1}{2} \frac{C_{D} \alpha}{m} \rho v^{2}
$$

where $v$ is the satellite velocity that, for a circular orbit, can be expressed as

$$
v=\sqrt{\frac{\mu}{\bar{a}}}
$$

The atmospheric density can be approximated using an exponential density model as

$$
\rho=\rho_{\text {ref }} \exp \left[-\frac{h_{\text {ref }}-h}{H}\right]
$$

For all analytical cases considered in this paper, the atmospheric density is modeled using the Committee on Space Research International Reference Atmosphere 1972 (CIRA-72) atmospheric model for 25-500 km and CIRA-72 with an exospheric temperature of $T_{\infty}=1000 \mathrm{~K}$ for $500-1000 \mathrm{~km}$, as presented in Refs. [39] and [40].

Once the acceleration caused by atmospheric friction has been calculated for the coast arc altitude using Eq. (36), the necessary $\Delta V$ to counteract the effect of atmospheric friction can be calculated as the required acceleration multiplied by the time of the coast arc:

$$
\Delta V_{2}=A_{\text {atm }} t_{2}
$$


This atmospheric friction compensation $\Delta V_{2}$ is added to $\Delta V_{\text {alt }}$ to give the total maneuver change in velocity $\Delta V_{\text {total }}$. It is of note that, because the atmospheric model used is divided into altitude bands for the calculation of atmospheric density, it is not possible to express Eq. (34) in terms of $\Delta V_{\text {total }}$ while maintaining a fully analytical solution. As such, the possible flyover instances are calculated as a function of $\Delta V_{\text {alt }}$. The corresponding $\Delta V_{2}$ is then calculated for each solution and included in the maneuver selection tradeoff.

\section{Comparison with Numerical Simulation}

A case study is used to compare the results produced by the general perturbation solution with those of a numerical simulation. The case selected considers a satellite in the same orbit as the International Space Station. The goal of the proposed mission is to reduce the revisit time of this satellite over Los Angeles, California in response to an earthquake in the region, for example. The orbital constants and satellite parameters for this and all other case studies are given in Tables $\underline{1}$ and 2 . The orbit parameters for this comparison case are given in Table 3 . The satellite propulsion system acceleration is calculated by assuming a $3 \mathrm{~kg}$ satellite (e.g., a 3 units (U) CubeSat $[41,42])$ equipped with the electrospray propulsion system developed by the Space Propulsion Laboratory of the Massachusetts Institute of Technology, which produces a nominal thrust of $350 \mu \mathrm{N}$ and has a specific impulse of $760 \mathrm{~s}$ [43-47]. For a $3 \mathrm{~kg} 3 \mathrm{U}$ CubeSat carrying $50 \mathrm{~g}$ of propellant, this equates to a maximum $\Delta V$ of $120 \mathrm{~m} / \mathrm{s}$, assuming a constant nominal thrust and a constant acceleration. This is a valid assumption because the propellant accounts for just $1.67 \%$ of the spacecraft wet mass and would see a change in acceleration of just $0.00198 \mathrm{~mm} / \mathrm{s}^{2}$ if the full propellant mass were used; this is a change of less than $1.7 \%$.

Figure 1 shows the distance from the POI to the subsatellite point over two days as calculated using Eq. (34) for an altitude-lowering maneuver using a $\Delta V_{\text {alt }}$ of $0-20 \mathrm{~m} / \mathrm{s}$. The times at which the POI will be in view for a given $\Delta V_{\text {alt }}$ are found by locating the minima of the analytical function and identifying those that fall within view of the satellite; these are shown as dots in Fig. 1. The solution is only plotted for feasible solutions for which the required $\Delta V$ can be produced in the allotted time. Following the paths of the constant maneuver time, it can be seen how the function varies with $\Delta V_{\text {alt }}$ and,

Table 1 Orbital constants.

\begin{tabular}{lccc}
\hline \hline Parameter & Symbol & Value & Units \\
\hline Gravitational parameter & $\mu$ & $3.986 \times 10^{14}$ & $\mathrm{~m}^{3} / \mathrm{s}^{2}$ \\
Mean radius of Earth & $R_{e}$ & $6.371 \times 10^{3}$ & $\mathrm{~km}$ \\
Coefficient of $J_{2}$ for Earth & $J_{2}$ & $1.0827 \times 10^{-3}$ & -- \\
Angular velocity of Earth & $\omega_{e}$ & $7.2921 \times 10^{-5}$ & $\mathrm{rad} / \mathrm{s}$ \\
\hline \hline
\end{tabular}

Table 2 Spacecraft parameters.

\begin{tabular}{lccc}
\hline \hline Parameter & Symbol & Value & Units \\
\hline Coefficient of drag & $C_{D}$ & 2.2 & -- \\
Satellite mass & $m$ & 3 & $\mathrm{~kg}$ \\
Satellite cross-sectional area & $\alpha$ & 0.03 & $\mathrm{~m}^{2}$ \\
\hline \hline
\end{tabular}

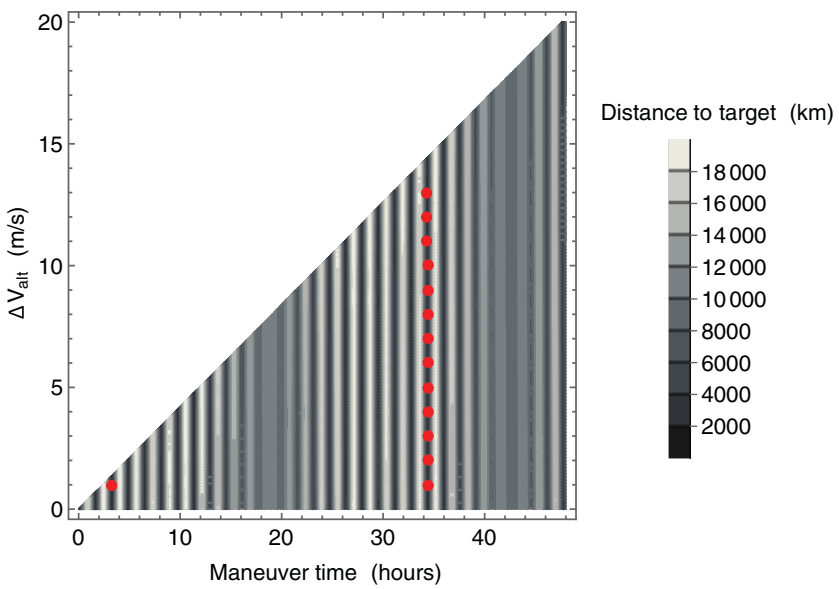

Fig. 1 Distance from subsatellite point to point of interest plotted as a function of maneuver time and $\Delta V_{\text {alt }}$. Dots indicate instances when target is in view of satellite.

conversely following a path of constant $\Delta V_{\text {alt }}$, it can be seen how the solution changes with increased maneuver time. In both cases, the solutions vary smoothly and cross the troughs and peaks of the surface that occur as a result of the satellite passage over one orbit period.

\section{A. Nonmaneuvering Satellite}

For a nonmaneuvering satellite (i.e., $\Delta V_{\text {alt }}=0 \mathrm{~m} / \mathrm{s}$ ), the distance from the SSP to the POI is shown in Fig. 2 for a 16-day period as calculated by the general perturbation method. The dashed horizontal line is drawn at half the swath width, taken to be $100 \mathrm{~km}$, indicating the distance at which the POI will be visible to the satellite, assuming a conical field of view. The times of each of the target flyovers and the distance of the SSP from the POI at that time are given in Table $\underline{4}$. This solution assumes that the satellite maintains a constant altitude by performing atmospheric friction compensation maneuvers throughout the 16-day period; this was calculated to require a $\Delta V$ of $3.22 \mathrm{~m} / \mathrm{s}$.

The numerical simulator used for comparison propagates the position of the spacecraft using a set of modified equinoctial elements [48] using an explicit variable-step-size Runge-Kutta formula of orders four and five formula: the Dormand-Prince pair [49]. The simulation includes an 18th-order tesseral model for the calculation of central body perturbations. Atmospheric friction is modeled using the U.S. Standard Atmosphere, 1976 model [50]; this is a more recent, and more detailed, atmospheric model than that used by the general perturbation solution. For all analyses, a relative and absolute error tolerance of $1 \times 10^{-12}$ and a solution refinement factor of 30 are used. The times at which the POI is found to be in view by the numerical simulation for a nonmaneuvering satellite are given in Table $\underline{4}$ with the corresponding distance to the target. The $\Delta V$ required for atmospheric friction compensation over this 16-day period was found to be $2.52 \mathrm{~m} / \mathrm{s}$; this is $20 \%$ less than the amount calculated using the analytical method.

Table 3 Los Angeles flyover mission parameters.

\begin{tabular}{lccc}
\hline \hline Parameter & Symbol & Value & Units \\
\hline Propulsion acceleration & $A$ & $\pm 1.1667 \times 10^{-4}$ & $\mathrm{~m} / \mathrm{s}^{2}$ \\
Inclination & $i$ & 51.64 & $\mathrm{deg}$ \\
Initial/final osculating semimajor axis & $a_{0}, a_{3}$ & 6773 & $\mathrm{~km}$ \\
Initial/final mean semimajor axis & $\bar{a}_{0}, \bar{a}_{3}$ & 6767 & $\mathrm{~km}$ \\
Initial AOL/RAAN & $u_{0}, \Omega_{0}$ & 0 & $\mathrm{deg}$ \\
Latitude of POI & $\delta_{\text {POI }}$ & 34.05 & $\mathrm{deg}$ \\
Longitude of POI & $\Psi_{\text {POI }}$ & -118.24 & $\mathrm{deg}$ \\
Epoch & -- & 1 Jan. 1990,00:00:00 hrs & - \\
Right ascension of Greenwich at epoch & $\Omega_{e t 0}$ & 100.39 & $\mathrm{deg}$ \\
Instrument swath & $s$ & 200 & $\mathrm{~km}$ \\
\hline \hline
\end{tabular}




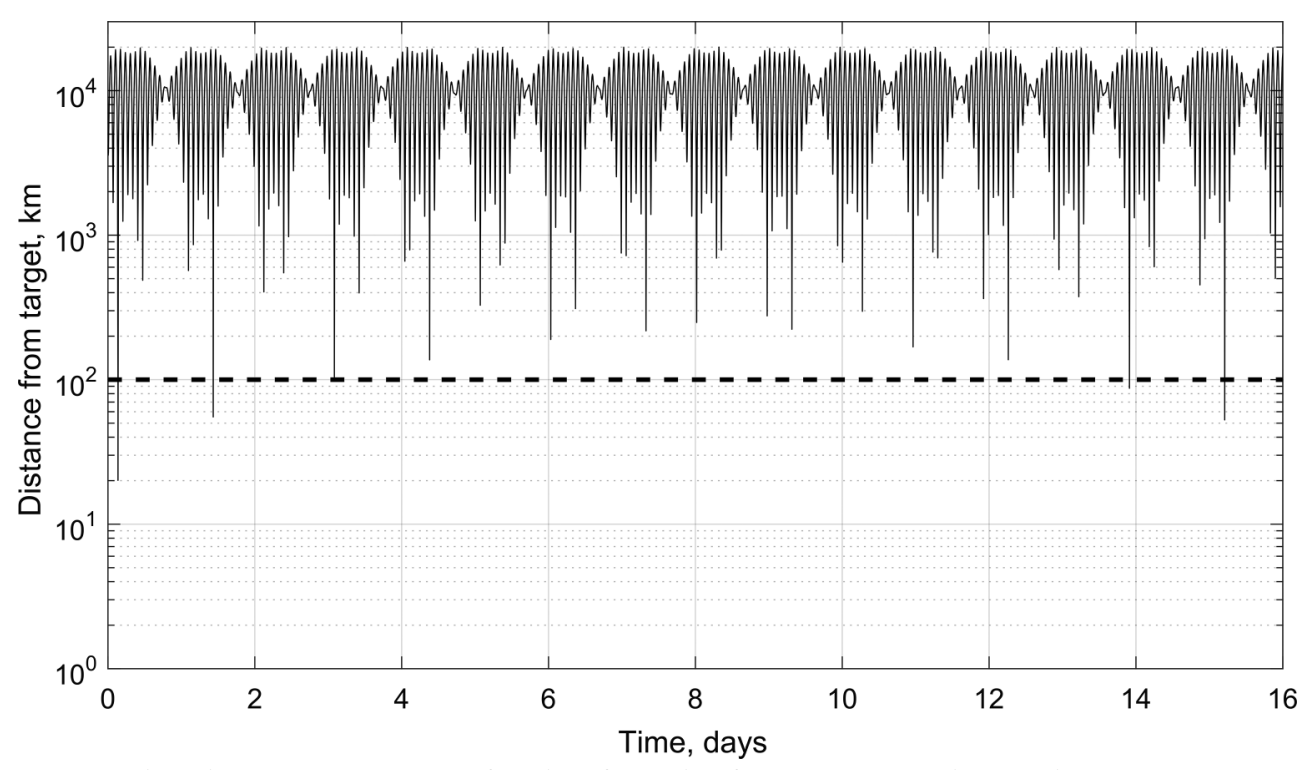

Fig. 2 Distance from subsatellite point to target plotted as a function of total time for a nonmaneuvering satellite as calculated by the analytical method.

The results in Table $\underline{4}$ show a close correlation between the general perturbation and numerical solutions. The greatest difference in flyover time identified by the analytical versus the numerical model is less than $3 \mathrm{~s}$, and the largest difference in the distance to the target at flyover is approximately $5 \mathrm{~km}$. It is of note that the analytical solution misses a pass at 13.91 days identified by the numerical simulation. This pass is at a distance of approximately $98 \mathrm{~km}$ from the POI, and so this is likely due to errors in the accuracy of the analytical model. As this pass is close to the edge of the field of view, it would be extremely short and of low quality. However, if it is desirable to ensure that passes close to the edge of the field are identified, a margin could be added to the swath width.

Figure 3 shows the haversine distance between the subsatellite points as calculated by the analytical and the numerical solutions over the same 16-day period as in Fig. 2. From these results, it is clear that the distance oscillates over time; this is due to the short-periodic variations in the orbital elements caused by $J_{2}$ and higher-order terms that are not included in the analytical solution. This is a key consideration when using this method and, indeed, all general perturbation methods because, due to the averaging of the disturbances over the orbit, the results will only be accurate after an integer number of orbit revolutions. Due to these oscillations, it is useful to consider the maximum difference and mean difference between the analytical and numerical methods. This is also shown in Fig. 3, where the mean and maximum values are calculated over a one-day period. These results show that the maximum difference gradually increases over time to just over $20 \mathrm{~km}$. The mean difference initially decreases before increasing to approximately $15 \mathrm{~km}$; this is likely the result of the long-periodic effects of $J_{2}$ and higher-order terms that are not accounted for in the analytical solution, as well as any discrepancies in the calculation of the secular effects of $J_{2}$ and atmospheric friction. These results indicate that, for a simulation of up to 16 days, an error bound of $25 \mathrm{~km}$ can be applied to the calculated distance to the target. It should be noted that this error bound is defined based on the specific case considered herein; cases with different orbit and satellite parameters may be subject to greater or lesser errors. It is of note that instance 4 from Table $\underline{4}$, which was not identified by the analytical solution, is just $2 \mathrm{~km}$ from the edge of the swath, and thus falls within this error range.

\section{B. Maneuvering Satellite}

The general perturbation method is now used to calculate the time required to fly over the target when the satellite is maneuvered using a given $\Delta V_{\text {alt }}$. Considering the results from Sec. III.A, it was decided that the maneuver should begin 1.433 days from epoch, which is at the time of the second viewing instance as calculated by the analytical method and given in Table 4 , with the aim of reducing the subsequent flyover time of the POI from the 13.8-day gap that would otherwise exist. This assumes that the passes at 3.08 and 13.91 days would be too short and too close to the edge of the swath width to be of value.

To compare with the numerical simulation, only a single maneuver is analyzed; the results for a range of values will be investigated in Sec. IV. An altitude-lowering maneuver using a $\Delta V_{\text {alt }}$ of $30 \mathrm{~m} / \mathrm{s}$ is arbitrarily selected for this, and the assumption is made that the satellite will return to its initial altitude at the end of the maneuver. Using a $\Delta V_{\text {alt }}$ of $30 \mathrm{~m} / \mathrm{s}$, the analytical solution identifies three possible maneuvers that would end with the satellite in view of the target. These are given in Table $\underline{5}$ with the corresponding distance from the SSP to the POI at closest approach. The shortest possible flyover time using $30 \mathrm{~m} / \mathrm{s}$ is found to be 5.06 days from epoch, shortening the time of flyover by more than 10 days when compared with the nonmaneuvering case. The atmospheric friction compensation $\Delta V_{2}$ required for this maneuver is found to be $0.21 \mathrm{~m} / \mathrm{s}$, giving a $\Delta V_{\text {total }}$ of $30.21 \mathrm{~m} / \mathrm{s}$.

The numerical simulator is used to investigate the solution found using the general perturbation method. To identify only any errors occurring during the maneuver, the simulation begins 1.433 days from epoch at viewing instance 2 from Table $\underline{4}$, as calculated by the analytical method. At this point, the three-phase maneuver is carried out, based on the solution found using the analytical method for flyover A, as given in Table 5. This consists of an initial thrusting phase to lower the orbit, lasting $35.71 \mathrm{~h}$, followed by a $15.54 \mathrm{~h}$ coast

Table 4 Comparison of flyover times and haversine distance to target for nonmaneuvering satellite calculated analytically versus numerically.

\begin{tabular}{|c|c|c|c|c|c|c|}
\hline \multirow[b]{2}{*}{ Viewing instance } & \multicolumn{2}{|r|}{ Analytical } & \multicolumn{2}{|r|}{ Numerical } & \multirow[b]{2}{*}{ Difference in flyover time, $\mathrm{s}$} & \multirow[b]{2}{*}{ Difference in distance, $\mathrm{km}$} \\
\hline & Time, days & Distance to target, $\mathrm{km}$ & Time, days & Distance to target, $\mathrm{km}$ & & \\
\hline 1 & 0.136 & 6.22 & 0.136 & 10.02 & -0.76 & -3.8 \\
\hline 2 & 1.433 & 69.74 & 1.433 & 65.50 & -0.27 & 4.24 \\
\hline 3 & 3.083 & 90.66 & 3.083 & 93.80 & -0.44 & -3.14 \\
\hline 4 & -- & -- & 13.91 & 98.39 & -- & -- \\
\hline 5 & 15.209 & 38.31 & 15.209 & 47.22 & 2.80 & -4.99 \\
\hline
\end{tabular}




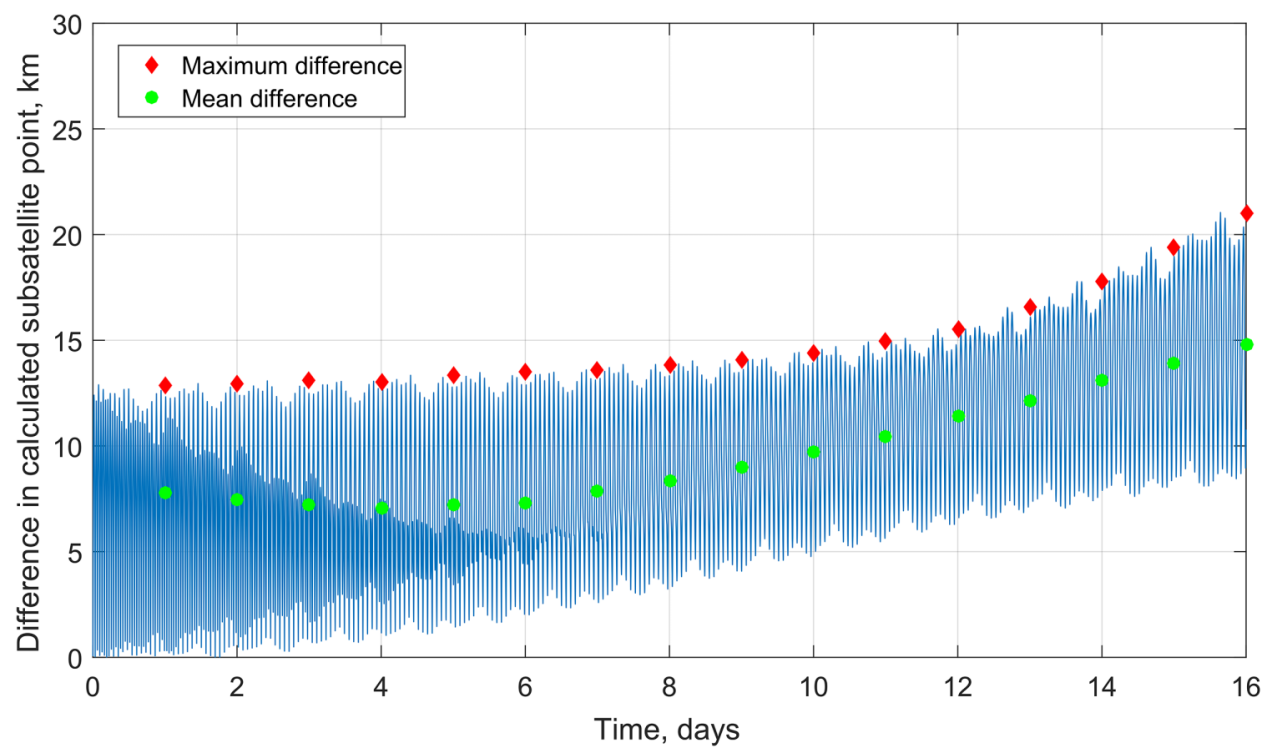

Fig. 3 Haversine distance between subsatellite points as calculated by analytical and numerical solutions for a nonmaneuvering satellite.

arc. Finally, a $35.71 \mathrm{~h}$ thrusting phase raises the orbit back to its initial altitude. The satellite is assumed to perform atmospheric friction compensation during the coast arc, as well as before and after the targeting maneuver.

Figure 4 shows the distance from the subsatellite point to the target as calculated using the haversine formula at each time step throughout the numerical simulation. The dashed horizontal line indicates the distance at which the POI will be visible to the satellite. The peak seen at 5.06 days from epoch corresponds to the pass that occurs at the end of the maneuver. This pass is found by the numerical solution to occur $32 \mathrm{~s}$ earlier than predicted by the analytical solution. The distance from the SSP to the target at this time is found to be $57.00 \mathrm{~km}$, which is a difference of $11.36 \mathrm{~km}$ when compared with the analytical solution. If atmospheric friction compensation is performed in the numerical simulation during the altitude change phases, in addition to the coast arc, the flyover occurs just $6.7 \mathrm{~s}$ after it is predicted by the analytical solution at a distance of $66.52 \mathrm{~km}$ from the POI, which is a difference of $2 \mathrm{~km}$ when compared with the analytical solution. These results imply that the effects of atmospheric drag are the largest contributor to the discrepancy in the time of flyover as predicted by the general perturbation method; however, the largest difference is very small at less than 1 min over a 3.6-day maneuver.

\section{Rapid Return Mission Case Study}

The case study presented in this section extends the scenario considered in Sec. III. As shown in Sec. III, if no maneuvers are performed, there is a 13.8-day gap between flyovers of Los Angeles after the second viewing at 1.433 days from epoch. It has been shown that an altitude-lowering maneuver using a $\Delta V_{\text {total }}$ of $30.21 \mathrm{~m} / \mathrm{s}$ can reduce the time between flyovers from 13.8 to 3.62 days. The same scenario is investigated here for a range of $\Delta V_{\text {total }}$ values and for both altitude-lowering and altitude-raising maneuvers. Using the general perturbation method, the shortest possible time in which a pass can be made over Los Angeles is calculated for a $\Delta V_{\text {alt }}$ range from $0-120 \mathrm{~m} / \mathrm{s}$ in increments of $1 \mathrm{~m} / \mathrm{s}$. The corresponding atmospheric friction compensation $\Delta V_{2}$ for each maneuver is then calculated

Table 5 Target flyover times for maneuvering satellite calculated analytically.

\begin{tabular}{lccc}
\hline \hline $\begin{array}{l}\text { Possible flyover } \\
\text { instance }\end{array}$ & $\begin{array}{c}\text { Time from } \\
\text { epoch, days }\end{array}$ & $\begin{array}{c}\text { Maneuver } \\
\text { time, days }\end{array}$ & $\begin{array}{c}\text { Distance from SSP to } \\
\text { POI, km }\end{array}$ \\
\hline A & 5.06 & 3.62 & 68.37 \\
B & 8.32 & 6.88 & 12.91 \\
C & 11.94 & 10.50 & 45.63 \\
\hline \hline
\end{tabular}

using Eq. (39) and added to give the change in velocity required for the full maneuver $\Delta V_{\text {total }}$. These results are shown in Fig. 5, where the circles indicate altitude-lowering maneuvers and the squares show altitude-raising maneuvers. The inset is a portion of Fig. $\underline{5}$ showing the maneuvers requiring a $\Delta V$ of less than $17 \mathrm{~m} / \mathrm{s}$ and approximately 1.65 days of maneuver time. Note that the time shown is the time taken for the maneuver, and not the time from epoch. The amount of $\Delta V_{2}$ required for atmospheric friction compensation is dependent on the maneuver selected, with those maneuvers requiring longer coast times at lower altitudes needing the greatest proportion; the largest $\Delta V_{2}$ required for the range of maneuvers considered is found to be $2.7 \mathrm{~m} / \mathrm{s}$. The results show that the minimum achievable flyover time is 1.65 days, or $39.59 \mathrm{~h}$, which is achieved with a $\Delta V_{\text {total }}$ of just $1.32 \mathrm{~m} / \mathrm{s}$ using an altitude-lowering maneuver. This gives a decrease in flyover time of almost 11 days when compared with the nonmaneuvering case.

Each maneuver option in Fig. $\underline{5}$ will have a corresponding minimum distance to the target at flyover that will define the payload look angle. This is shown in Fig. 6 for the solutions requiring a maneuver time of approximately 1.6 days and a $\Delta V_{\text {alt }}$ of $1-17 \mathrm{~m} / \mathrm{s}$. Note that, in order to produce a continuous contour, Fig. 6 is plotted in terms of $\Delta V_{\text {alt }}$, and so it does not include the additional $\bar{\Delta} V_{2}$ required for atmospheric friction compensation. The shortest possible maneuver, which is an altitude-lowering maneuver requiring $39.59 \mathrm{~h}$ and a $\Delta V_{\text {total }}$ of $1.32 \mathrm{~m} / \mathrm{s}$, will have a distance to target at flyover of greater than $90 \mathrm{~km}$; this is seen in the bottom left of Fig. $\underline{6}$. The other set of contours corresponds to the possible altitude-raising maneuvers. The minimum time altitude-raising maneuver takes $39.6 \mathrm{~h}$ and requires a $\Delta V_{\text {total }}$ of $1.31 \mathrm{~m} / \mathrm{s}$; however, it has a distance to target at flyover of greater than $80 \mathrm{~km}$. Increasing the $\Delta V_{\text {alt }}$ used increases the required maneuver time but also reduces the minimum distance to the target at flyover. The minimum distance to the target that can be obtained using maneuvers with a $\Delta V_{\text {alt }}$ of less than $17 \mathrm{~m} / \mathrm{s}$ is approximately $30 \mathrm{~km}$; however, there are other maneuver options shown in Fig. 5 that will enable a direct flyover of the target at the cost of higher $\Delta \bar{V}$ and maneuver time. In addition, it may be possible to obtain a direct flyover by altering the thrust profile of the lower $\Delta V$ maneuvers using the analytical solution as an initial guess. These insights obtained from the solution space can be extremely valuable to an operator, allowing for an informed tradeoff between mission goals, and are difficult to gain through numerical methods alone.

A maneuver using a $\Delta V_{\text {alt }}$ of $12 \mathrm{~m} / \mathrm{s}$, corresponding to a $\Delta V_{\text {total }}$ of $12.08 \mathrm{~m} / \mathrm{s}$ and predicting a distance to target at flyover of $34 \mathrm{~km}$, is selected as a compromise between $\Delta V_{\text {total }}$, the flyover time, and the look angle at flyover. This maneuver is analyzed using the numerical propagator described in Sec. III. The results from the analytical 


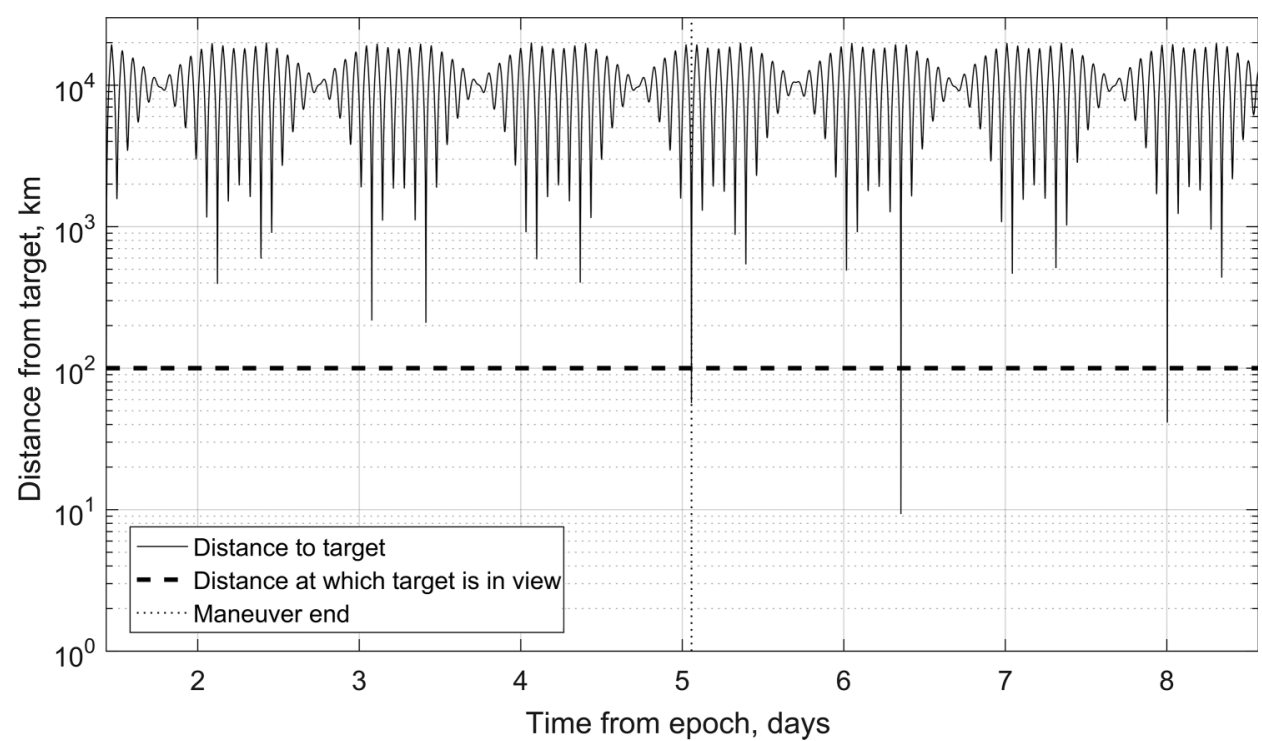

Fig. 4 Distance from subsatellite point to target as calculated by numerical simulation. Maneuver begins at 1.433 days, following the solution for flyover A from Table $\underline{5}$ given by the analytical method.

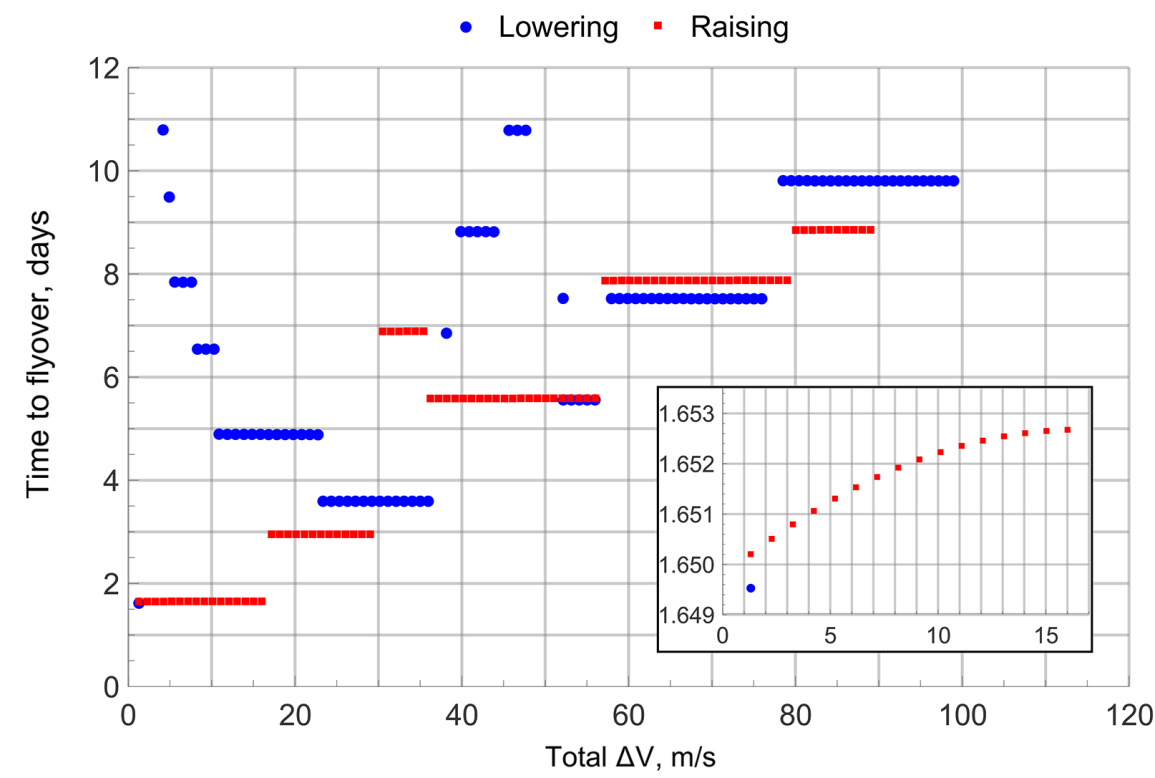

Fig. 5 Shortest time to flyover for a given $\Delta V_{\text {total }}$ as calculated by the analytical method. Inset shows maneuvers requiring $\Delta V<17 \mathrm{~m} / \mathrm{s}$ and approximately 1.65 days of maneuver time.

method are used to define the maneuver to be carried out, and atmospheric friction compensation is assumed to be performed during phase 2, as well as before and after the maneuver. The satellite begins the three-phase altitude-raising maneuver 1.433 days from

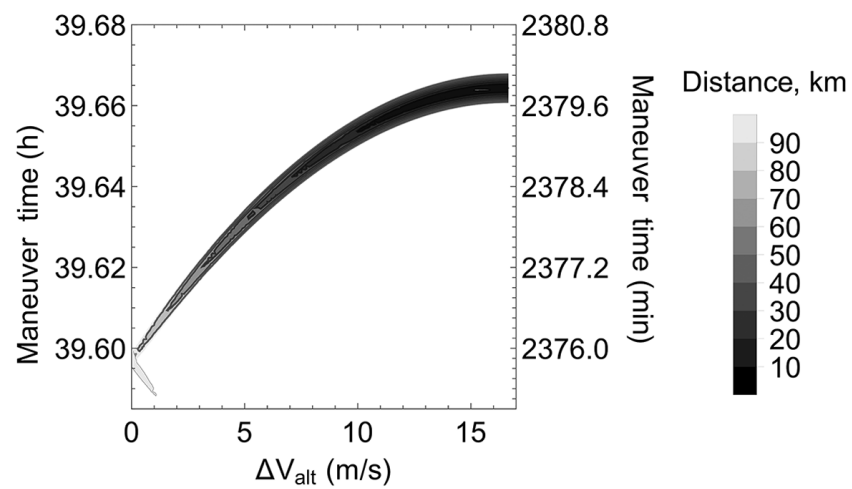

Fig. 6 Minimum distance to target for selected solutions as a function of maneuver time and required $\Delta V_{\text {alt }}$. epoch. Figure 7 shows the distance from the subsatellite point to the target as calculated using the haversine formula at each time step throughout this mission. As before, the dashed horizontal line indicates the distance at which the POI will be visible to the satellite. A peak can be seen in Fig. 7 corresponding to a pass that occurs at 3.09 days from epoch. This pass occurs just $4 \mathrm{~s}$ earlier than predicted by the analytical solution, with the distance from the SSP to the target at this time found to be $38 \mathrm{~km}$; this is $4 \mathrm{~km}$ greater than predicted by the analytical solution. The total $\Delta V$ required is found to be $12.06 \mathrm{~m} / \mathrm{s}$, including that required for atmospheric friction compensation. This gives a difference of just $0.02 \mathrm{~m} / \mathrm{s}$ when compared with the general perturbation solution.

\section{Fire Response Case Study}

The case study presented in this section considers a constellation tasked with fire detection and subsequent maneuvering to provide targeted coverage of fire outbreaks. This scenario is based on the "reconfigurable constellation" concept of operations, in which the Earth observing constellation has two operational modes that it can maneuver between $[20,51,52]$. The first is a global observation mode 


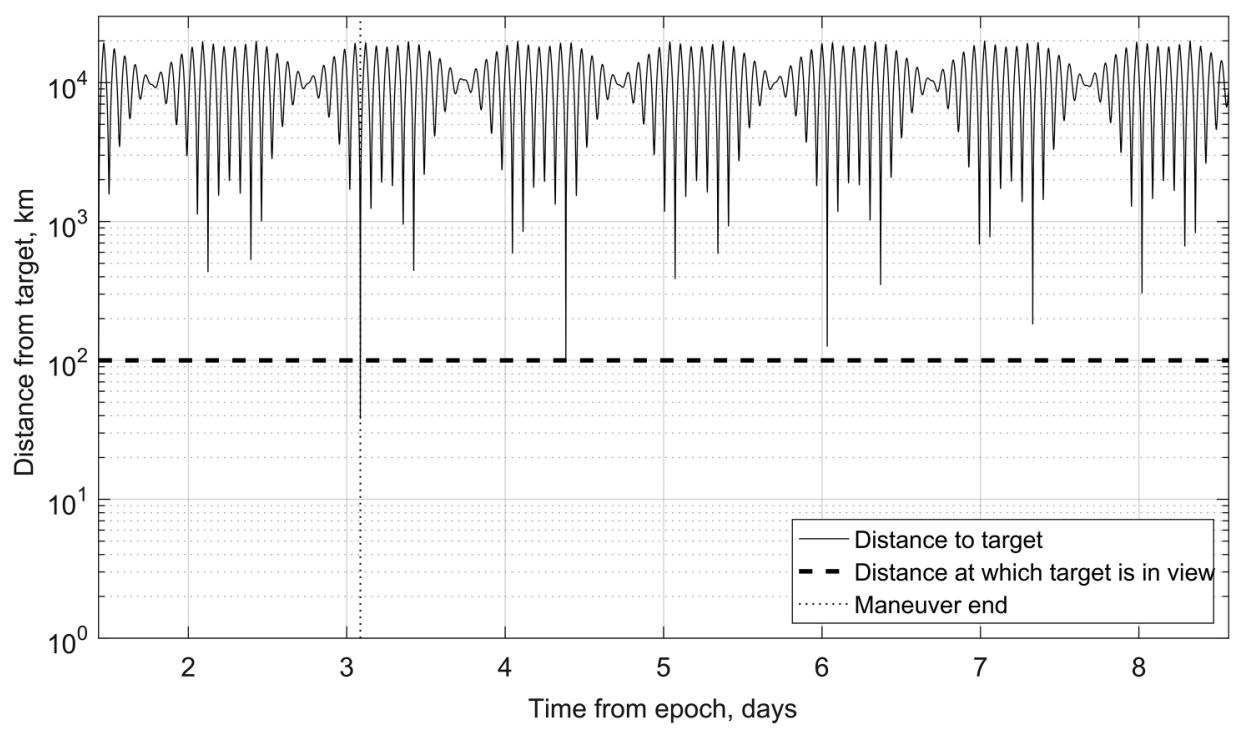

Fig. 7 Distance from subsatellite point to target as calculated by numerical simulation. Altitude-raising maneuver begins at 1.433 days following a $12.08 \mathrm{~m} / \mathrm{s}$ maneuver as calculated by the analytical method.

(GOM), in which the satellites are spread out to provide even coverage of the observation region. The second mode is the regional observation mode (ROM), in which some of the satellites are moved into repeating ground track (RGT) orbits over a point of interest to provide improved coverage of the target. For the proposed mission, the constellation in the GOM would be used to detect the outbreak of fire and then would transition to the ROM to provide more frequent revisits of the affected area. Once targeted coverage is no longer required, the constellation returns to the GOM to continue global observations.

Sequential fire outbreaks in four different locations are considered to assess the effectiveness of a responsive constellation to provide increased coverage of a range of latitudes. The areas considered are Cairngorms National Park in Scotland; Yosemite National Park in California; Agulhas National Park in South Africa; and Lagunas de Montebello National Park in Mexico. The constellation proposed for this study comprises 24 satellites in four orbit planes at an inclination of $60 \mathrm{deg}$. For this inclination, a repeat ground track of 15 orbits per day, giving two daily overflights of the target, requires a mean altitude of $513.087 \mathrm{~km}$, as calculated for a circular orbit using the method described in Refs. [20] and [53]. The initial mean altitude of the GOM constellation is arbitrarily selected as $542.857 \mathrm{~km}$. From this altitude, a satellite requires a minimum $\Delta V$ of $17 \mathrm{~m} / \mathrm{s}$ to reach the ROM orbit altitude. The parameters of the constellation are given in Table 6 . The proposed swath width of $50 \mathrm{~km}$ is based on a mission design proposing the use of a constellation of CubeSats for fire detection [54]. The mission is assumed to use $3 \mathrm{U}$ CubeSats equipped with electrospray propulsion providing a $\Delta V$ of $120 \mathrm{~m} / \mathrm{s}$, as in Secs. III and IV, and the constants in Table $\underline{1}$ and parameters in Table $\underline{2}$ are used.

To transition between the GOM and ROM, two satellites per plane will be maneuvered into repeating ground tracks (i.e., eight satellites in total), with one satellite providing coverage of the target region on

Table 6 Fire response constellation mission parameters.

\begin{tabular}{lcc}
\hline \hline Parameter & Value & Units \\
\hline Propulsion acceleration & $\pm 1.1667 \times 10^{-4}$ & $\mathrm{~m} / \mathrm{s}^{2}$ \\
Inclination & 60 & $\mathrm{deg}$ \\
GOM initial osculating/mean altitude & $550 / 542.857$ & $\mathrm{~km}$ \\
ROM initial osculating/mean altitude & $520.261 / 513.087$ & $\mathrm{~km}$ \\
Number of orbit planes & 4 & -- \\
Number of satellites per plane & 6 & - \\
RAAN spacing between orbit planes & 90 & $\mathrm{deg}$ \\
In-plane spacing between satellites & 60 & $\mathrm{deg}$ \\
Epoch & $1 \mathrm{Jan} .1990,00: 00: 00 \mathrm{hrs}$ & - \\
Right ascension of Greenwich at epoch & 100.39 & $\mathrm{deg}$ \\
Instrument swath & 50 & $\mathrm{~km}$ \\
\hline \hline
\end{tabular}

the upward pass and the other providing coverage on the downward pass. Moving additional satellites provides limited benefit because there are only two positions in each RGT orbit plane that provide a flyover of the target; thus, the maneuvering of more satellites would result in multiple satellites positioned very close together, providing overlapping coverage and limited increased utility. The satellites remaining in the GOM orbit are not rephased to provide even coverage from this altitude; however, they will still provide incidental coverage of the target.

For all targets, the region of interest is taken to be a rectangle encompassing the park with the parameters given in Table 7. The fire events occur sequentially in each region as follows: 1) Cäirngorms, 2) Yosemite, 3) Agulhas, and 4) Lagunas de Montebello. For each outbreak, the constellation will maneuver to the ROM and then remain in the ROM for seven days before transitioning back to the GOM. The constellation then remains in the GOM for seven days before the next fire is detected. The exception to this is the transition between targeting Yosemite and Agulhas; in this case, the fire in Agulhas is assumed to be detected while the constellation is still targeting Yosemite, and so a direct transition from ROM to ROM occurs.

\section{A. Method}

To decide which satellites should be maneuvered during each reconfiguration, and what form these maneuvers should take, each satellite is analyzed using the general perturbation method described in Sec. II, producing a list of all possible maneuvers that each satellite could perform that would conclude with the satellite over the target region and in a repeating ground track orbit. This is done for a $\Delta V_{\text {alt }}$ range of $17-120 \mathrm{~m} / \mathrm{s}$ in increments of $1 \mathrm{~m} / \mathrm{s}$ for up to 10 days of total maneuver time, and it considers both altitude-raising and altitudelowering maneuvers. The satellites are assumed to perform atmospheric friction compensation at all times, except when performing altitude change maneuvers; this applies to the satellites in both the GOM and ROM.

To select the "best" maneuvers from the full solution set, the solutions are grouped first by orbit plane and then by whether they will view the target on an upward or downward pass. A simple additive multiattribute utility function is then applied to produce a single utility score for each solution $[55,56]$. The solution in each grouping with the highest utility function is selected. In some cases, where the difference in utility function between satellites was small, a judgment was made to select the preferred solution; this was done to bring the desired outcome in line with what a mission operator would likely select while avoiding the need to fine tune the utility function, which is outside the scope of this paper.

A simple additive multiattribute utility function can be described by 
Table 7 National Park locations

\begin{tabular}{lccrrr}
\hline \hline Region of interest & Minimum latitude & Maximum latitude & Minimum longitude & Maximum longitude & Units \\
\hline Cairngorms & 56.58 & 57.66 & -4.64 & -2.65 & $\mathrm{deg}$ \\
Yosemite & 37.49 & 38.18 & -119.89 & -119.20 & $\mathrm{deg}$ \\
Agulhas & -34.83 & -34.68 & 19.63 & 20.02 & $\mathrm{deg}$ \\
Lagunas de Montebello & 16.07 & 16.12 & -91.74 & -91.63 & $\mathrm{deg}$ \\
\hline \hline
\end{tabular}

Table 8 Summary of coverage available to target regions in a seven-day period for both a static and responsive constellation

\begin{tabular}{|c|c|c|c|c|c|c|c|c|}
\hline & \multicolumn{2}{|c|}{ Cairngorms } & \multicolumn{2}{|c|}{ Yosemite } & \multicolumn{2}{|c|}{ Agulhas } & \multicolumn{2}{|c|}{$\begin{array}{l}\text { Lagunas de } \\
\text { Montebello }\end{array}$} \\
\hline & Static & Responsive & Static & Responsive & Static & Responsive & Static & Responsive \\
\hline Average coverage of region, min & 2.8 & 4.5 & 1.0 & 4.4 & 1.0 & 5.6 & 0.64 & 6.9 \\
\hline Peak local coverage, min & 3.2 & 7.7 & 1.1 & 6.6 & 1.1 & 7.2 & 0.7 & 7.0 \\
\hline Average revisit time, $\mathrm{h}$ & 5.6 & 3.9 & 16.1 & 3.8 & 16.7 & 3.0 & 23.8 & 2.8 \\
\hline Maximum local revisit time, $\mathrm{h}$ & 15.5 & 17.7 & 56.8 & 23.7 & 62.3 & 11.7 & 48.6 & 4.7 \\
\hline
\end{tabular}

$$
U(X)=\sum_{n=1}^{N} k_{n} U_{n}\left(X_{n}\right)
$$

where $X_{n}$ is a single attribute, $U_{n}\left(X_{n}\right)$ is the utility function of a single attribute, $k_{n}$ is a weighting factor for a single attribute, $U(X)$ is the multiattribute utility function, and $N$ is the number of attributes to be considered. The weighting factors determine the relative weighting to be given to each attribute. For this scenario, the attributes selected are the $\Delta V_{\text {total }}$ required for the maneuver, the maneuver time, and the distance to the center of the region of interest at the end of the maneuver. Each of these is normalized against the range of possible values of the attribute to give a utility between zero and one. That is,

$$
U_{n}=\frac{X_{\text {worst }}-X_{n}}{X_{\text {worst }}-X_{\text {ideal }}}
$$

where $X_{\text {worst }}$ is the worst possible value of the attribute, and $X_{\text {ideal }}$ is the best possible value. A relative weighting is applied with $\Delta V_{\text {total }}$ taken as the normal value, and so $k_{\Delta V}=1$, and relative weightings are given to the maneuver time and distance to target, respectively, as $k_{\text {time }}=3$ and $k_{\text {distance }}=0.5$ to prioritize the maneuver time over the other attributes. It is of note that this criteria selection will tend to focus coverage on the center of the POI; selecting different attribute criteria could allow for coverage to be evenly spread across the region, for example.

Once the fire has been dealt with, it is assumed that the constellation will return to the GOM to continue global observation. This is considered a nonurgent maneuver, and so the minimum $\Delta V_{\text {alt }}$ possible is used, with the required satellite in-plane phasing being achieved by the satellite remaining in the RGT orbit for as long as is necessary before maneuvering. In this case, there is essentially no phase 1 maneuver; instead, the satellite coasts as in phase 2 before raising its altitude to return to the GOM constellation. To calculate the required maneuver time for each satellite, a simplified version of the general perturbation method is used in which the time and $\Delta V$ for phase 1 are both set to zero. Taking any satellite in the same plane and at the GOM altitude as a reference, the desired difference in the final AOLs of the maneuvering satellite and the reference satellite can be specified, and thus the total required maneuver time calculated to achieve the desired spacing using the minimum $\Delta V_{\text {alt }}$ of $17 \mathrm{~m} / \mathrm{s}$. All possible maneuvers are investigated and the solutions selected to give the shortest total maneuver time for each plane. On return to the GOM using this method, the satellite spacing within each orbit plane will return to $60 \mathrm{deg}$; however, the RAAN of the maneuvered satellites will have a slight variation from the other satellites. This could be readjusted by raising the altitude of these satellites above the GOM altitude and allowing them to drift in the RAAN before rephasing. For this study, the orbit planes were not adjusted and the largest difference in the RAAN between two satellites in the same plane was approximately $2.5 \mathrm{deg}$ at the end of the mission.
When maneuvering to the target of Agulhas National Park, the constellation is assumed to begin maneuvering directly from observing Yosemite with no transition to the GOM. The same method is used to select the satellites to maneuver; in this case, five of the satellites selected to maneuver were already in RGT orbits over Yosemite, whereas the other three selected were in the higher GOM orbit. This leaves three satellites in RGT orbits over Yosemite that should be returned to the GOM. As before, this was done using the minimum possible $\Delta V_{\text {alt }}$ of $17 \mathrm{~m} / \mathrm{s}$ with the required satellite inplane phasing being achieved by the satellite remaining in the RGT orbit for as long as required.

\section{B. Results}

To assess the improvement in the coverage and revisit time that could be achieved through the use of the proposed responsive constellation, a comparison is made with a static constellation of 24 satellites inclined at $60 \mathrm{deg}$ and at a mean altitude of $542.857 \mathrm{~km}$; this is the same as the initial GOM constellation. The coverage available for both the static constellation and the responsive constellation is analyzed over a one-week period using a simple orbit propagator that includes only the secular effects of $J_{2}$. A summary of these results is given in Table $\underline{8}$, where the average coverage and revisit time refers to the mean value across the entire region of interest. Peak coverage refers to the maximum coverage available to any single location within the region of interest. The maximum local revisit time refers to the maximum time that any single location within the region is not viewed.

Figures 8-11 visually depict the coverage available to each region from the static constellation and the responsive constellation over a one-week period. From these results, it is clear that, in all cases, the responsive constellation provides greater coverage than the static constellation. A more significant improvement in coverage is seen for those targets at lower latitudes because the coverage available to them from the static constellation is lower when compared to targets at the upper latitudes of the satellites' visible region. For example, the responsive constellation provides average regional coverage to the Cairngorms of approximately 1.6 times that available from the static constellation, whereas the Lagunas de Montebello receives more than 10 times greater coverage from the responsive constellation as compared to the static constellation. Also of interest, as seen in Table 8 , is that the revisit time provided by the responsive constellation is consistent for all latitudes, with a revisit time of between 2.5 and $4.0 \mathrm{~h}$ for each of the regions considered. This is in contrast to the static constellation that has a longer revisit time at lower latitudes.

Table 9 summarizes the entire mission. The mission takes just less than 82 days, assuming seven days for each ROM and GOM phase, and requires a total $\Delta V$ of $1019 \mathrm{~m} / \mathrm{s}$, including that required for

Map data: Google, INEGI, AfriGIS (Pty) Ltd., www.google.com/maps, [retrieved 08 November 2017]. 

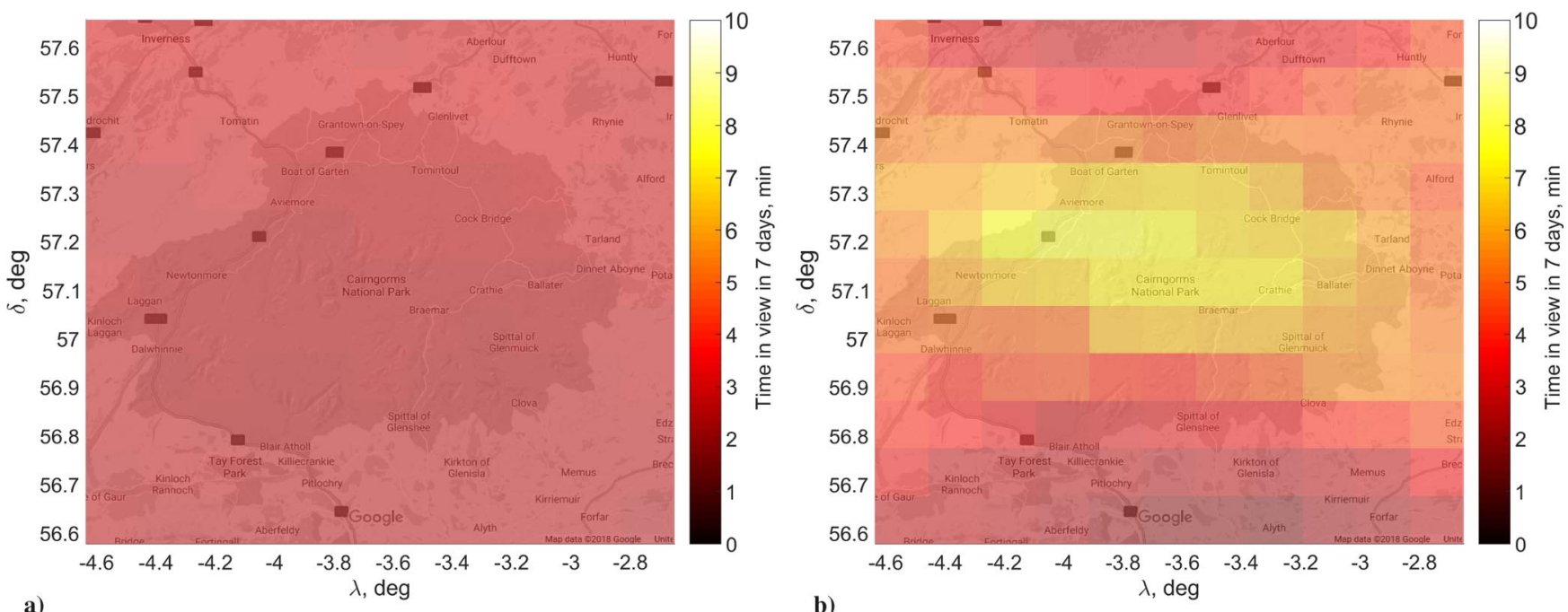

Fig. 8 Total time a region of Cairngorms was seen in a one-week period for a) the static constellation, and b) the responsive constellation. (See footnote $\ddagger$.)
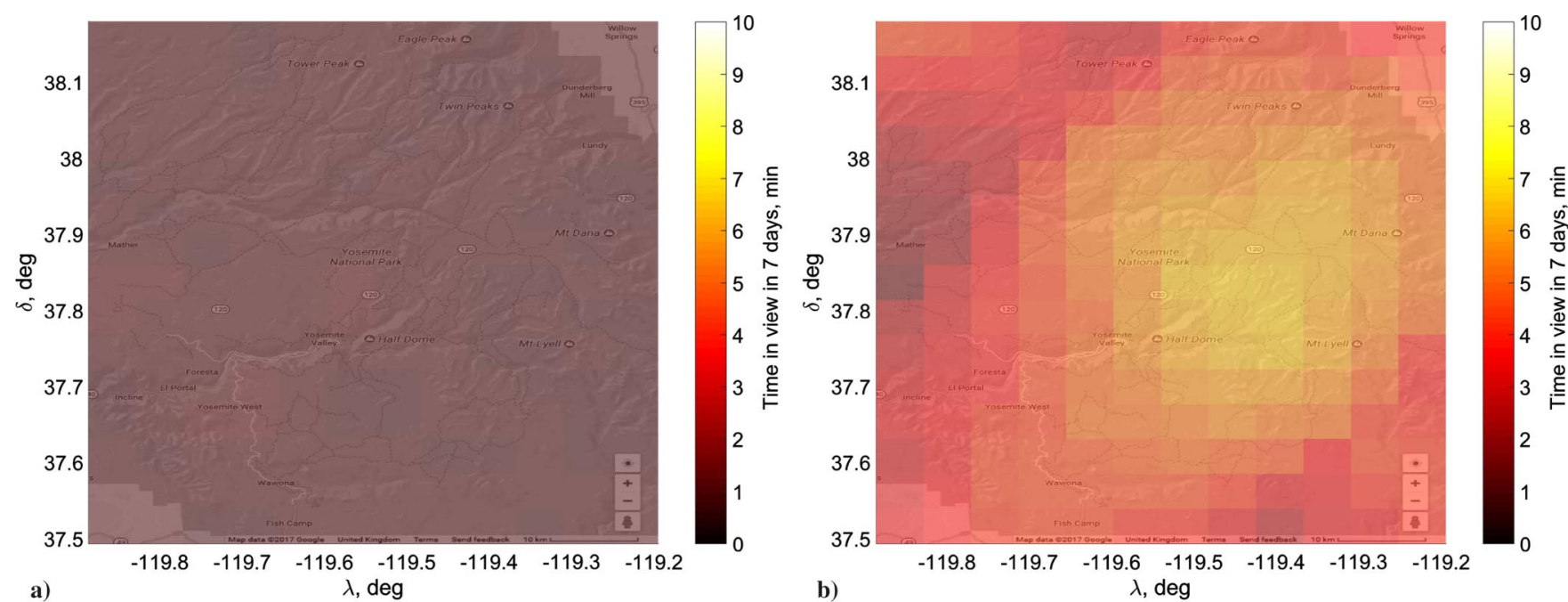

Fig. 9 Total time a region of Yosemite was seen in a one-week period for a) the static constellation, and b) the responsive constellation. (See footnote $\ddagger$.)
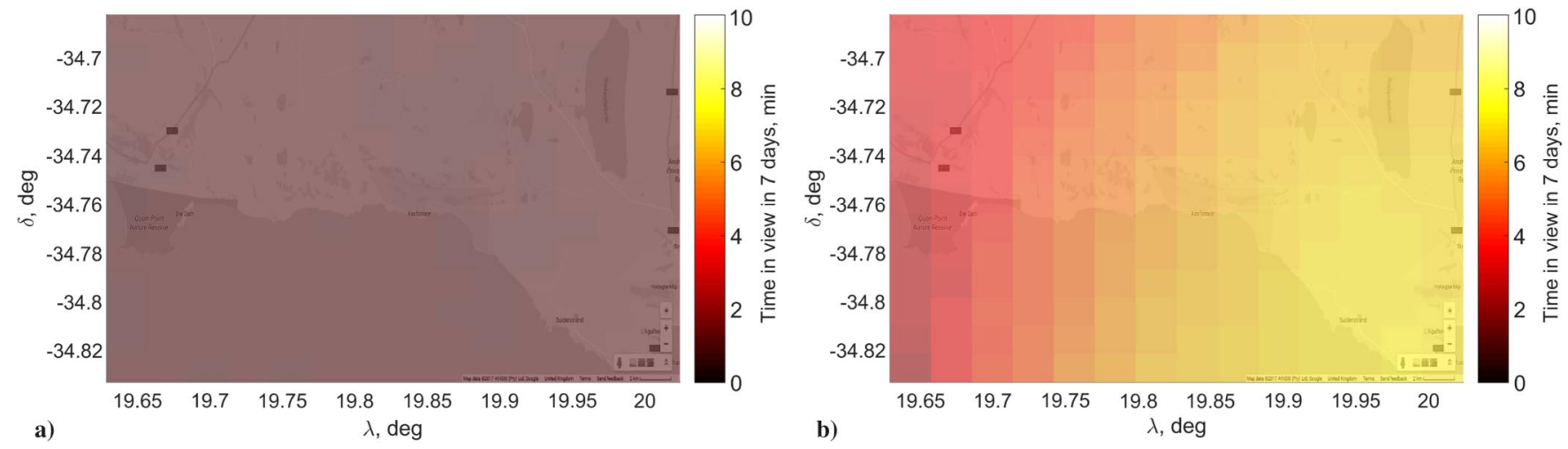

Fig. 10 Total time a region of Agulhas was seen in a one-week period for a) the static constellation, and b) the responsive constellation. (See footnote.+$)$

atmospheric friction compensation throughout; this is just $35 \%$ of the total $\Delta V$ available across the constellation. Satellite 13 uses the largest proportion of propellant, requiring a $\Delta V$ of almost $109 \mathrm{~m} / \mathrm{s}$. Satellites $2,3,9,11,18$, and 20 all perform no altitude-changing maneuvers throughout the mission and require a $\Delta V$ of just $1.6 \mathrm{~m} / \mathrm{s}$ for atmospheric friction compensation. The average $\Delta V$ used across all satellites is $42 \mathrm{~m} / \mathrm{s}$; thus, it is likely that the constellation could perform numerous further reconfigurations before all propellant would be depleted. The standard deviation of the propellant usage across all satellites is $30.04 \mathrm{~m} / \mathrm{s}$.
It is possible to balance propellant usage by including the satellites' remaining propellant in the maneuver selection utility function. To do this, an additional attribute term $U_{\mathrm{fb}}$ was added to the utility function to account for the propellant remaining on board each satellite. This was included in Eq. (41) with a weighting of $k_{\mathrm{fb}}=2$. In this scenario, the first two reconfigurations to target the Cairngorms and Yosemite are the same as for the case with no propellant balancing. However, beyond this point, the scenario changes due to the new utility function. The mission takes 1.5 days longer and uses $161 \mathrm{~m} / \mathrm{s}$ more $\Delta V$; however, this is still just $41 \%$ of the total available 

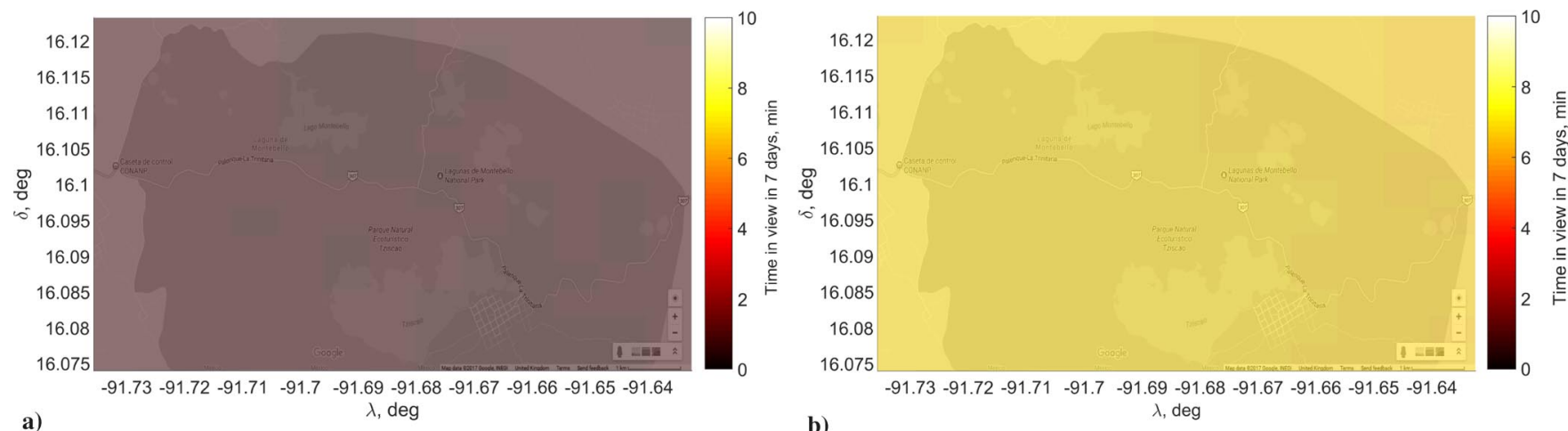

Fig. 11 Total time a region of Lagunas de Montebello was seen in a one-week period for a) the static constellation, and b) the responsive constellation (See footnote :.)

Table 9 Fire response mission $\Delta V$ and time required for each section.

\begin{tabular}{lcccc}
\hline \hline Section & Section time, days & Total time, days & Section $\Delta V, \mathrm{~m} / \mathrm{s}$ & Total $\Delta V, \mathrm{~m} / \mathrm{s}$ \\
\hline Start & 0.00 & 0.00 & 0 & 0 \\
Maneuver to target Cairngorms & 2.87 & 2.87 & 144.25 & 144.25 \\
Observing Cairngorms in ROM & 7.00 & 9.87 & 4.00 & 148.25 \\
Return to GOM & 10.37 & 20.24 & 140.74 & 288.99 \\
Time in GOM & 7.00 & 27.24 & 3.36 & 292.35 \\
Maneuver to target Yosemite & 4.72 & 31.96 & 181.96 & 474.31 \\
Observing Yosemite in ROM & 7.00 & 38.96 & 4.00 & 478.31 \\
Maneuver to target Agulhas & 7.92 & 46.88 & 187.94 & 666.25 \\
Observing Agulhas in ROM & 7.00 & 53.88 & 4.00 & 670.25 \\
Return to GOM & 9.59 & 63.47 & 140.82 & 811.07 \\
Time in GOM & 7.00 & 70.47 & 3.36 & 814.43 \\
Maneuver to target Chiapas & 4.01 & 74.48 & 200.77 & 1015.20 \\
Observing Chiapas in ROM & 7.00 & 81.48 & 4.00 & 1019.20 \\
\hline \hline
\end{tabular}

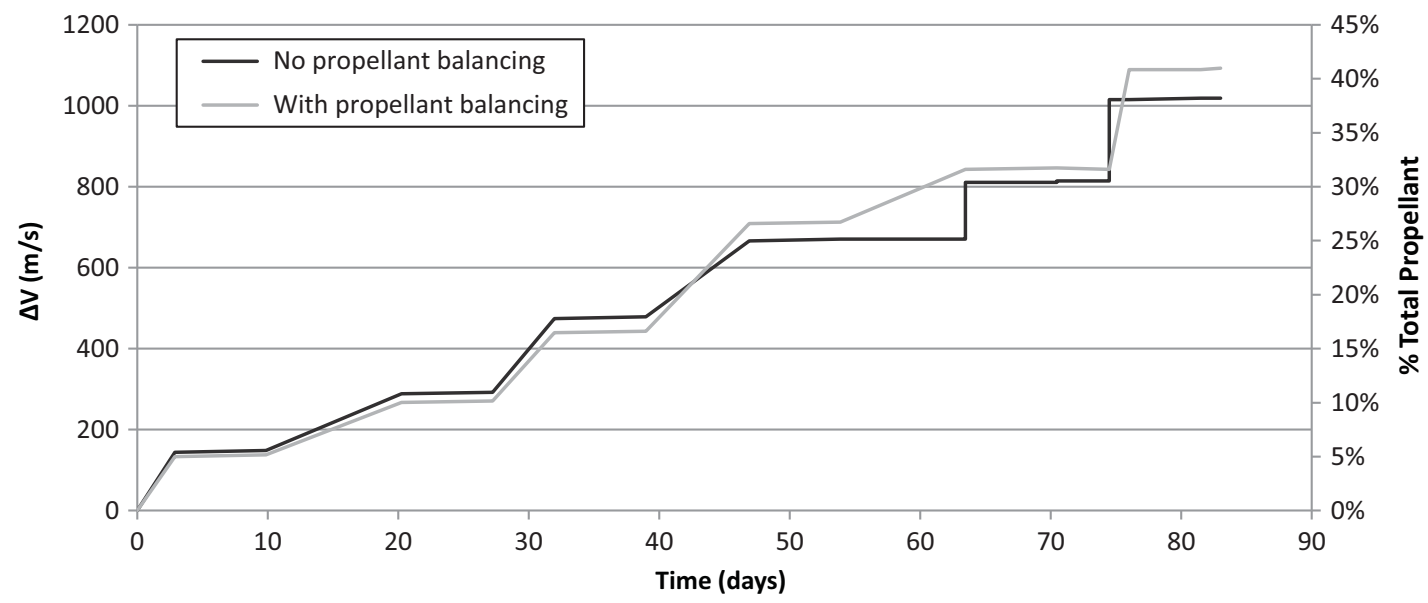

Fig. 12 Total $\Delta V$ used over time for the fire response mission for cases with and without propellant balancing.

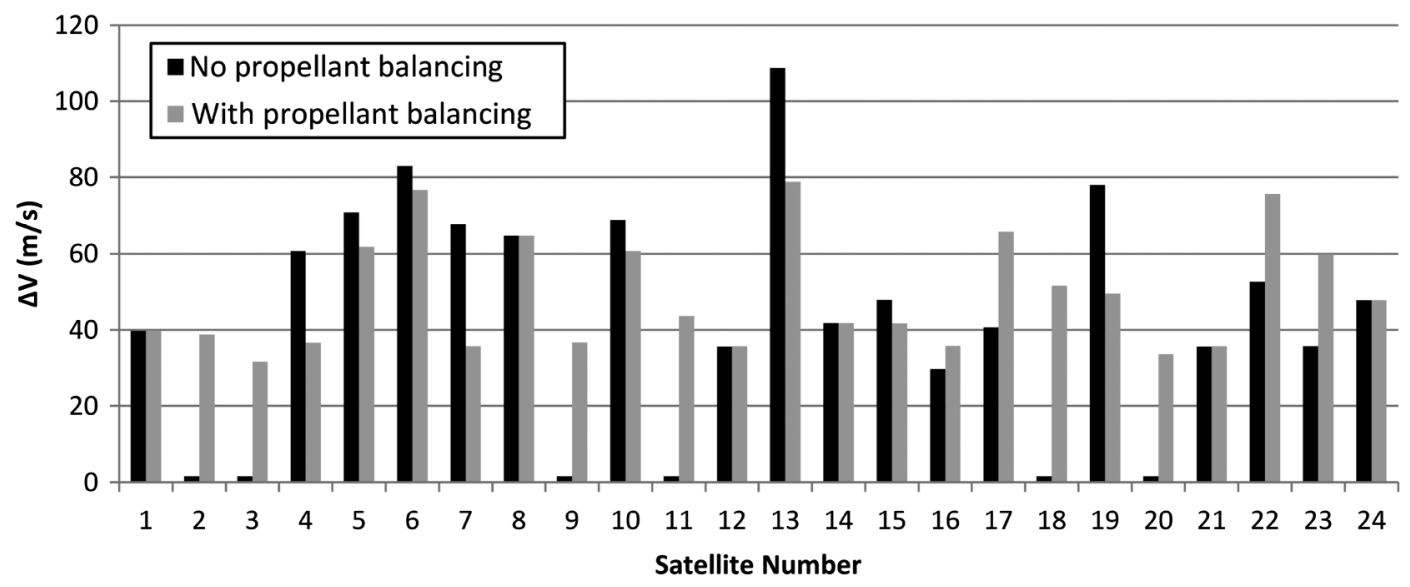

Fig. $13 \Delta V$ used by each satellite in the fire response mission for cases with and without propellant balancing. 
across the constellation. The standard deviation of propellant usage across all satellites in this case is reduced to $15.03 \mathrm{~m} / \mathrm{s}$, with all satellites having maneuvered; and the largest amount of $\Delta V$ used by a single satellite is $79 \mathrm{~m} / \mathrm{s}$, which was used by satellite 13 . The total cumulative $\Delta V$ used over time for the cases with and without propellant balancing is shown in Fig. 12. Figure 13 shows the total $\Delta V$ used by each satellite for both cases. It is clear that, although the inclusion of propellant balancing uses a greater $\Delta V$ overall, it does result in a more even spread of propellant use across the individual satellites.

\section{Conclusions}

A general perturbation method can be used to solve the presented, restricted low-thrust Lambert rendezvous problem and gain an insight into the capabilities of a maneuverable satellite, or satellites, that would be difficult to achieve using numerical methods alone. Complex scenarios including numerous satellites and thousands of possible maneuvers can be analyzed extremely quickly and without iteration; for the cases considered herein, a single maneuver can be analyzed in $0.5 \mathrm{~s}$ using Wolfram Mathematica 11.3 running on a desktop computer with an Intel Core i7-4790 CPU and 8 GB of RAM.
By providing all possible solutions, the method grants the mission designer the ability to select, from the full solution set, the maneuver, or maneuvers, that best meet the mission goals. This removes reliance on numerical optimizers to calculate suitable maneuver profiles because these cannot guarantee that the optimal solution is found. Responsive missions carried out using existing technology can provide significant improvements in the volume and frequency of data collection when compared with traditional, static satellite missions. A small satellite equipped with low-thrust propulsion is capable of reducing the revisit time to a target by more than $85 \%$ for less than a $20 \mathrm{~m} / \mathrm{s}$ velocity change. The choice of maneuver requires a three-way tradeoff between the change in velocity required, the maneuver time, and the look angle to target. A constellation of satellites can provide increased coverage of between 1.6 and 10 times to regions of interest when compared with a static, global coverage constellation by maneuvering into repeating ground track orbits over the target. The revisit time of this constellation postreconfiguration is more uniform across latitudes than a traditional static constellation that will provide more frequent revisits to higher-latitude targets. Applying propellant balancing across the constellation increases the overall velocity change required for the mission but more evenly distributes the propellant usage across the satellites.

\section{Appendix: Equations for Right Ascension of Ascending Node and Argument of Latitude Change}

$$
\begin{aligned}
& \Delta \Omega_{1}=\frac{1}{7560 \sqrt{2} A J_{2} R_{e}^{2}} \cot (i) \csc ^{7}(i)\left\{\frac { 1 } { ( 3 \gamma - 2 a _ { 0 } ^ { 2 } ) ^ { 3 } } \sqrt { \frac { \mu } { a _ { 0 } ^ { 3 } } } a _ { 0 } \sqrt { \frac { \mu \operatorname { c s c } ^ { 6 } ( i ) a _ { 0 } ^ { 3 } } { ( 2 \operatorname { c s c } ^ { 2 } ( i ) a _ { 0 } ^ { 2 } - 3 J _ { 2 } R _ { e } ^ { 2 } ) ^ { 3 } } } \left[432 \gamma(129-236 \cos (2 i)+171 \cos (4 i)) a_{0}^{6}\right.\right. \\
& -64(129-236 \cos (2 i)+171 \cos (4 i)) a_{0}^{8}-2268(57-108 \cos (2 i)+83 \cos (4 i)) \gamma^{2} a_{0}^{4}+2835(43-84 \cos (2 i)+73 \cos (4 i)) \gamma^{3} a_{0}^{2} \\
& \left.+102,060(1+3 \cos (2 i)) \gamma^{4} \sin ^{2}(i)\right]+\frac{\mu^{4} a_{0}^{3}}{\beta\left\{\left[32 \mu^{2} \csc ^{2}(i) a_{0}^{2} / \beta^{2}\right]-3 J_{2} R_{e}^{2}\right\}^{3}} 4 \mu \csc ^{6}(i) \sqrt{\frac{\beta^{3}}{\mu^{2} a_{0}^{3}}} a_{0} \sqrt{\frac{\beta^{3}\left[-3 \gamma+\left(32 \mu^{2} a_{0}^{2} / \beta^{2}\right)\right]^{3}}{\beta^{4}}} \\
& -\frac{419,4304 \mu^{8}(129-236 \cos (2 i)+171 \cos (4 i)) a_{0}^{8}}{\beta^{8}}-\frac{580,608 \mu^{4}(57-108 \cos (2 i)+83 \cos (4 i)) a_{0}^{4} \gamma^{2}}{\beta^{4}} \\
& \left.\left.+\frac{45,360 \mu^{2}(43-84 \cos (2 i)+73 \cos (4 i)) a_{0}^{2} \gamma^{3}}{\beta^{2}}+102,060(1+3 \cos (2 i)) \gamma^{4} \sin ^{2}(i)+\frac{1,769,472 \gamma \mu^{6}(129-236 \cos (2 i)+171 \cos (4 i)) a_{0}^{6}}{\beta^{6}}\right]\right\}
\end{aligned}
$$$$
\begin{aligned}
& \Delta \Omega_{2}=-\frac{3 \cos (i) J_{2} R_{e}^{2}}{2\left[\left(4 \mu a_{0} / \beta\right)-\left(3 \gamma \beta / 8 \mu a_{0}\right)\right]^{2}}\left\{\frac{\left.\sqrt{\mu}\left[-640 a_{0}^{2}\left(\mu a_{0} / \beta\right)^{5 / 2}+96\left(-2+3 \sin ^{2}(i)\right)\left(\mu a_{0} / \beta\right)^{5 / 2} J_{2} R_{e}^{2}+a_{0}^{5 / 2}\left(320 \mu^{2} a_{0}^{2} / \beta^{2}\right)+3\left(2-3 \sin ^{2}(i)\right) J_{2} R_{e}^{2}\right)\right]}{640 A a_{0}^{5 / 2}\left(\mu a_{0} / \beta\right)^{5 / 2}}\right. \\
& \left.-\frac{\sqrt{\mu}\left[-\left(320 \mu^{2} a_{0}^{2} a_{3}^{5 / 2} / \beta^{2}\right)+3\left(-2+3 \sin ^{2}(i)\right) a_{3}^{5 / 2} J_{2} R_{e}^{2}+32\left(\mu a_{0} / \beta\right)^{5 / 2}\left(20 a_{3}^{2}+3\left(2-3 \sin ^{2}(i)\right) J_{2} R_{e}^{2}\right)\right]}{640 A\left(\mu a_{0} / \beta\right)^{5 / 2} a_{3}^{5 / 2}}+t_{t}\right\} \\
& \times \sqrt{\frac{\mu}{\left[\left(4 \mu a_{0} / \beta\right)-\left(3 \gamma \beta / 8 \mu a_{0}\right)\right]^{3}}}\left[1+\frac{3\left\{1-\left[3 \sin ^{2}(i) / 2\right]\right\} J_{2} R_{e}^{2}}{2\left(4 \mu a_{0} / \beta\right)-\left(3 \gamma \beta / 8 \mu a_{0}\right)^{2}}\right]
\end{aligned}
$$

$$
\begin{aligned}
& \Delta \Omega_{3}=-\frac{1}{7560 \sqrt{2} A J_{2} R_{e}^{2}} \cot (i) \csc ^{7}(i)\left\{\frac{1}{\left(2 \csc ^{2}(i) a_{3}^{2}-3 J_{2} R_{e}^{2}\right)^{3}} \csc ^{6}(i) \sqrt{\frac{\mu}{a_{3}^{3}}} a_{3} \sqrt{\frac{\mu a_{3}^{3}}{\left(-3 \gamma+2 a_{3}^{2}\right)^{3}}}[432 \gamma(129-236 \cos (2 i)\right. \\
& +171 \cos (4 i)) a_{3}^{6}-64(129-236 \cos (2 i)+171 \cos (4 i)) a_{3}^{8}-2268(57-108 \cos (2 i)+83 \cos (4 i)) a_{3}^{4} \gamma^{2} \\
& \left.+2835(43-84 \cos (2 i)+73 \cos (4 i)) a_{3}^{2} \gamma^{3}+102,060(1+3 \cos (2 i)) \sin ^{2}(i) \gamma^{4}\right] \\
& +\frac{1}{\beta\left[3 \gamma-\left(32 \mu^{2} a_{0}^{2} / \beta^{2}\right)\right]^{3}} 4 \mu \sqrt{\frac{\beta^{3}}{\mu^{2} a_{0}^{3}}} a_{0} \sqrt{\frac{\mu^{4} \csc ^{6}(i) a_{0}^{3}}{\beta^{3}\left\{\left[32 \mu^{2} \csc ^{2}(i) a_{0}^{2} / \beta^{2}\right]-3 J_{2} R_{e}^{2}\right\}^{3}}}\left[-\frac{4,194,304 \mu^{8}(129-236 \cos (2 i)+171 \cos (4 i)) a_{0}^{8}}{\beta^{8}}\right. \\
& \quad-\frac{580,608 \mu^{4}(57-108 \cos (2 i)+83 \cos (4 i)) a_{0}^{4} \gamma^{2}}{\beta^{4}}+\frac{45,360 \mu^{2}(43-84 \cos (2 i)+73 \cos (4 i)) a_{0}^{2} \gamma^{3}}{\beta^{2}} \\
& \left.\left.+102,060(1+3 \cos (2 i)) \sin ^{2}(i) \gamma^{4}+\frac{1,769,472 \gamma \mu^{6}(129-236 \cos (2 i)+171 \cos (4 i)) a_{0}^{6}}{\beta^{6}}\right]\right\}
\end{aligned}
$$




$$
\begin{aligned}
& \Delta u_{1}=-\frac{1}{918,540 \sqrt{2} A \sqrt{\left(\mu / a_{0}^{3}\right)} a_{0}^{7} J_{2}^{6} R_{e}^{12}} \csc ^{12}(i)\left[\frac{\mu a_{0}^{3}}{\left(-3 \gamma+2 a_{0}^{2}\right)^{3}}\right]^{3 / 2}\left\{18,600,435(1+3 \cos (2 i)) \sin ^{18}(i) J_{2}^{10} R_{e}^{20}\right. \\
& +229,635 \sin ^{12}(i) a_{0}^{2} J_{2}^{6} R_{e}^{12}\left[-216 \gamma^{3}+3(1+3 \cos (2 i)) J_{2} R_{e}^{2}\left(-180 \gamma^{2}+3 \gamma \kappa\right)\right]-2835 \sin ^{6}(i) a_{0}^{4} J_{2}^{3} R_{e}^{6}\left[3(1+3 \cos (2 i)) J_{2} R_{e}^{2}\right. \\
& \left.\times\left(9 \zeta \gamma^{2}+432 \kappa \gamma^{3}-25920 \gamma^{4}\right)-216 \gamma^{3}\left(144 \gamma^{2}+3 \gamma \kappa\right)\right]+2268 \sin ^{4}(i) a_{0}^{6} J_{2}^{2} R_{e}^{4}\left[3(1+3 \cos (2 i)) J_{2} R_{e}^{2}\left(9 \zeta \gamma^{2}+459 \kappa \gamma^{3}-25920 \gamma^{4}\right)\right. \\
& \left.-6 \gamma\left(5184 \gamma^{4}+108 \gamma^{3} \kappa+9 \gamma^{2} \zeta\right)\right]+64 a_{0}^{10}\left[3(1+3 \cos (2 i)) J_{2} R_{e}^{2}\left(18 \zeta \gamma^{2}+918 \kappa \gamma^{3}-51435 \gamma^{4}\right)-6 \gamma\left(13203 \gamma^{4}+351 \gamma^{3} \kappa+18 \gamma^{2} \zeta\right)\right] \\
& \left.\left.-432 \gamma a_{0}^{8}\left[-6 \gamma\left(10368 \gamma^{4}+351 \gamma^{3} \kappa+18 \gamma^{2} \zeta\right)+3(1+3 \cos (2 i)) J_{2} R_{e}^{2}\left(-51435 \gamma^{4}+918 \gamma^{3} \kappa+18 \gamma^{2} \zeta\right)\right]\right\}\right) \\
& +\frac{1}{3,674,160 \sqrt{2} A \mu^{7} \sqrt{\left(\beta^{3} / \mu^{2} a_{0}^{3}\right)} a_{0}^{7} J_{2}^{6} R_{e}^{12}} \beta^{7} \csc ^{12}(i)\left[\frac{\mu^{4} a_{0}^{3}}{\beta^{3}\left[-3 \gamma+\left(32 \mu^{2} a_{0}^{2} / \beta^{2}\right)\right]^{3}}\right]^{3 / 2}\left\{18,600,435(1+3 \cos (2 i)) \sin ^{18}(i) J_{2}^{10} R_{e}^{20}\right. \\
& +\frac{3,674,160 \mu^{2} \sin ^{12}(i) a_{0}^{2} J_{2}^{6} R_{e}^{12}\left[-216 \gamma^{3}+3(1+3 \cos (2 i)) J_{2} R_{e}^{2}\left(-180 \gamma^{2}+3 \gamma \kappa\right)\right]}{\beta^{2}}-\frac{1}{\beta^{4}} 725,760 \mu^{4} \sin ^{6}(i) a_{0}^{4} J_{2}^{3} R_{e}^{6} \\
& {\left[3(1+3 \cos (2 i)) J_{2} R_{e}^{2}\left(9 \zeta \gamma^{2}+432 \kappa \gamma^{3}-25920 \gamma^{4}\right)-216 \gamma^{3}\left(144 \gamma^{2}+3 \gamma \kappa\right)\right]+\frac{1}{\beta^{6}} 9,289,728 \mu^{6} \sin ^{4}(i) a_{0}^{6} J_{2}^{2} R_{e}^{4}} \\
& {\left[3(1+3 \cos (2 i)) J_{2} R_{e}^{2}\left(9 \zeta \gamma^{2}+459 \kappa \gamma^{3}-25920 \gamma^{4}\right)-6 \gamma\left(5184 \gamma^{4}+108 \gamma^{3} \kappa+9 \gamma^{2} \zeta\right)\right]+\frac{1}{\beta^{10}} 67,108,864 \mu^{10}} \\
& a_{0}^{10}\left[3(1+3 \cos (2 i)) J_{2} R_{e}^{2}\left(18 \zeta \gamma^{2}+918 \kappa \gamma^{3}-51435 \gamma^{4}\right)-6 \gamma\left(13203 \gamma^{4}+351 \gamma^{3} \kappa+18 \gamma^{2} \zeta\right)\right] \\
& \left.-\frac{28,311,552 \gamma \mu^{8} a_{0}^{8}\left[-6 \gamma\left(10368 \gamma^{4}+351 \gamma^{3} \kappa+18 \gamma^{2} \zeta\right)+3(1+3 \cos (2 i)) J_{2} R_{e}^{2}\left(-51435 \gamma^{4}+918 \gamma^{3} \kappa+18 \gamma^{2} \zeta\right)\right]}{\beta^{8}}\right\} \\
& \Delta u_{2}=\left\{\sqrt{\frac{\mu}{\left[-\left(3 \beta \gamma / 8 \mu a_{0}\right)+\left(4 \mu a_{0} / \beta\right)\right]^{3}}}\left[1+\frac{3\left[1-\left(3 \sin ^{2}(i) / 2\right)\right] J_{2} R_{e}^{2}}{2\left[-\left(3 \beta \gamma / 8 \mu a_{0}\right)+\left(4 \mu a_{0} / \beta\right)\right]^{2}}\right]\right. \\
& \left.+\frac{3\left[2-\left(5 \sin ^{2}(i) / 2\right)\right] \sqrt{\frac{\mu}{\left[-\left(3 \beta \gamma / 8 \mu a_{0}\right)+\left(4 \mu a_{0} / \beta\right)\right]^{3}}} J_{2} R_{e}^{2}\left(1+\left(3\left[1-\left(3 \sin ^{2}(i) / 2\right)\right] J_{2} R_{e}^{2} / 2\left[-\left(3 \beta \gamma / 8 \mu a_{0}\right)+\left(4 \mu a_{0} / \beta\right)\right]^{2}\right)\right)}{2\left[-\left(3 \beta \gamma / 8 \mu a_{0}\right)+\left(4 \mu a_{0} / \beta\right)\right]^{2}}\right\} \\
& \left\{-\frac{1}{2 A}\left[-\frac{9 \gamma \sqrt{\mu}}{10 a_{0}^{5 / 2}}+\frac{2 \sqrt{\mu}}{\sqrt{a_{0}}}+\frac{9 \gamma \sqrt{\mu}}{320\left(\mu a_{0} / \beta\right)^{5 / 2}}-\frac{\sqrt{\mu}}{\sqrt{\left(\mu a_{0} / \beta\right)}}+\frac{3 \sqrt{\mu} J_{2} R_{e}^{2}}{5 a_{0}^{5 / 2}}-\frac{3 \sqrt{\mu} J_{2} R_{e}^{2}}{160\left(\mu a_{0} / \beta\right)^{5 / 2}}\right]\right. \\
& \left.+\frac{1}{2 A}\left[-\frac{9 \gamma \sqrt{\mu}}{320\left(\mu a_{0} / \beta\right)^{5 / 2}}+\frac{\sqrt{\mu}}{\sqrt{\left(\mu a_{0} / \beta\right)}}+\frac{9 \gamma \sqrt{\mu}}{10 a_{3}^{5 / 2}}-\frac{2 \sqrt{\mu}}{\sqrt{a_{3}}}+\frac{3 \sqrt{\mu} J_{2} R_{e}^{2}}{160\left(\mu a_{0} / \beta\right)^{5 / 2}}-\frac{3 \sqrt{\mu} J_{2} R_{e}^{2}}{5 a_{3}^{5 / 2}}\right]+t_{t}\right\}
\end{aligned}
$$$$
\Delta u_{3}=-\frac{1}{918,540 \sqrt{2} A \sqrt{\left(\mu / a_{3}^{3}\right)} a_{3}^{7} J_{2}^{6} R_{e}^{12}} \csc ^{12}(i)\left[\frac{\mu a_{3}^{3}}{\left(-3 \gamma+2 a_{3}^{2}\right)^{3}}\right]^{3 / 2}\left\{18,600,435(1+3 \cos (2 i)) \sin ^{18}(i) J_{2}^{10} R_{e}^{20}\right.
$$$$
+229,635 \sin ^{12}(i) a_{3}^{2} J_{2}^{6} R_{e}^{12}\left[-216 \gamma^{3}+3(1+3 \cos (2 i)) J_{2} R_{e}^{2}\left(-180 \gamma^{2}+3 \gamma \kappa\right)\right]
$$$$
-2835 \sin ^{6}(i) a_{3}^{4} J_{2}^{3} R_{e}^{6}\left[3(1+3 \cos (2 i)) J_{2} R_{e}^{2}\left(9 \zeta \gamma^{2}+432 \kappa \gamma^{3}-25920 \gamma^{4}\right)-216 \gamma^{3}\left(144 \gamma^{2}+3 \gamma \kappa\right)\right]
$$$$
+2268 \sin ^{4}(i) a_{3}^{6} J_{2}^{2} R_{e}^{4}\left[3(1+3 \cos (2 i)) J_{2} R_{e}^{2}\left(9 \zeta \gamma^{2}+459 \kappa \gamma^{3}-25920 \gamma^{4}\right)-6 \gamma\left(5184 \gamma^{4}+108 \gamma^{3} \kappa+9 \gamma^{2} \zeta\right)\right]
$$$$
+64 a_{3}^{10}\left(3(1+3 \cos (2 i)) J_{2} R_{e}^{2}\left(18 \zeta \gamma^{2}+918 \kappa \gamma^{3}-51435 \gamma^{4}\right)-6 \gamma\left(13203 \gamma^{4}+351 \gamma^{3} \kappa+18 \gamma^{2} \zeta\right)\right)
$$$$
\left.-432 \gamma a_{3}^{8}\left[-6 \gamma\left(10368 \gamma^{4}+351 \gamma^{3} \kappa+18 \gamma^{2} \zeta\right)+3(1+3 \cos (2 i)) J_{2} R_{e}^{2}\left(-51435 \gamma^{4}+918 \gamma^{3} \kappa+18 \gamma^{2} \zeta\right)\right]\right\}
$$$$
+\frac{1}{3,674,160 \sqrt{2} A \mu^{7} \sqrt{\left(\beta^{3} / \mu^{2} a_{0}^{3}\right)} a_{0}^{7} J_{2}^{6} R_{e}^{12}} \beta^{7} \csc ^{12}(i)\left[\frac{\mu^{4} a_{0}^{3}}{\beta^{3}\left[-3 \gamma+\left(32 \mu^{2} a_{0}^{2} / \beta^{2}\right)\right]^{3}}\right]^{3 / 2}\left\{18,600,435(1+3 \cos (2 i)) \sin ^{18}(i) J_{2}^{10} R_{e}^{20}\right.
$$$$
+\frac{1}{\beta^{2}} 3,674,160 \mu^{2} \sin ^{12}(i) a_{0}^{2} J_{2}^{6} R_{e}^{12}\left[-216 \gamma^{3}+3(1+3 \cos (2 i)) J_{2} R_{e}^{2}\left(-180 \gamma^{2}+3 \gamma \kappa\right)\right]
$$$$
-\frac{1}{\beta^{4}} 725,760 \mu^{4} \sin ^{6}(i) a_{0}^{4} J_{2}^{3} R_{e}^{6}\left[3(1+3 \cos (2 i)) J_{2} R_{e}^{2}\left(9 \zeta \gamma^{2}+432 \kappa \gamma^{3}-25920 \gamma^{4}\right)-216 \gamma^{3}\left(144 \gamma^{2}+3 \gamma \kappa\right)\right]
$$$$
+\frac{1}{\beta^{6}} 9,289,728 \mu^{6} \sin ^{4}(i) a_{0}^{6} J_{2}^{2} R_{e}^{4}\left[3(1+3 \cos (2 i)) J_{2} R_{e}^{2}\left(9 \zeta \gamma^{2}+459 \kappa \gamma^{3}-25920 \gamma^{4}\right)-6 \gamma\left(5184 \gamma^{4}+108 \gamma^{3} \kappa+9 \gamma^{2} \zeta\right)\right]
$$$$
+\frac{1}{\beta^{10}} 67,108,864 \mu^{10} a_{0}^{10}\left[3(1+3 \cos (2 i)) J_{2} R_{e}^{2}\left(18 \zeta \gamma^{2}+918 \kappa \gamma^{3}-51435 \gamma^{4}\right)-6 \gamma\left(13203 \gamma^{4}+351 \gamma^{3} \kappa+18 \gamma^{2} \zeta\right)\right]
$$$$
\left.28,311,552 \gamma \mu^{8} a_{0}^{8}\left[-6 \gamma\left(10368 \gamma^{4}+351 \gamma^{3} \kappa+18 \gamma^{2} \zeta\right)+3(1+3 \cos (2 i)) J_{2} R_{e}^{2}\left(-51435 \gamma^{4}+918 \gamma^{3} \kappa+18 \gamma^{2} \zeta\right)\right]\right\}
$$ 
where

$$
\begin{aligned}
& \gamma=J_{2} R_{e}^{2} \sin ^{2}(i) \\
& \zeta=72 J_{2}^{2} R_{e}^{4} \sin ^{4}(i)+9 J_{2}^{2} R_{e}^{4}\left(7 \sin ^{2}(i)-2\right)\left(9 \sin ^{2}(i)-4\right) \\
& \kappa=3 J_{2} R_{e}^{2}\left(7 \sin ^{2}(i)-2\right)+3 J_{2} R_{e}^{2}\left(9 \sin ^{2}(i)-4\right)
\end{aligned}
$$

and, for the case where the satellite altitude is lowered in phase 1,

$$
\beta=a_{0}\left(\sqrt{\frac{\mu}{a_{3}}}+\Delta V_{\text {alt }}\right)\left(2 \sqrt{\frac{\mu}{a_{0}}}+\sqrt{\frac{\mu}{a_{3}}}+\Delta V_{\text {alt }}\right)+\mu
$$

whereas for the case in which the satellite altitude is raised in phase 1 ,

$$
\beta=a_{0}\left(\sqrt{\frac{\mu}{a_{3}}}-\Delta V_{\text {alt }}\right)\left(2 \sqrt{\frac{\mu}{a_{0}}}+\sqrt{\frac{\mu}{a_{3}}}-\Delta V_{\text {alt }}\right)+\mu
$$

\section{Acknowledgments}

This research was supported by the Engineering and Physical Sciences Research Council (grant number EP/M506643/1) and the Air Force Office of Scientific Research (grant number FA9550-18-10147). The collaboration with the Massachusetts Institute of Technology was supported by the Fulbright-Schuman Awards scheme. There are no data underpinning this publication; all results can be fully reproduced using the methods described in this paper. The authors would like to acknowledge the support of Paulo Lozano, David Miller, and David Krejci of the Massachusetts Institute of Technology.

\section{References}

[1] Sandau, R., Brieß, K., and D’Errico, M., "Small Satellites for Global Coverage: Potential and Limits," International Society for Photogrammetry and Remote Sensing Journal of Photogrammetry and Remote Sensing, Vol. 65, No. 6, 2010, pp. 492-504. doi:10.1016/j.isprsiprs.2010.09.003

[2] Tyc, G., Tulip, J., Schulten, D., Krischke, M., and Oxfort, M., "The RapidEye Mission Design," Acta Astronautica, Vol. 56, Nos. 1-2, 2005, pp. 213-219.

doi:10.1016/j.actaastro.2004.09.029

[3] da Silva Curiel, A., Boland, L., Cooksley, J., Bekhti, M., Stephens, P., Sun, W., and Sweeting, M., "First Results from the Disaster Monitoring Constellation (DMC)," Acta Astronautica, Vol. 56, Nos. 1-2, 2005, pp. 261-271.

doi:10.1016/j.actaastro.2004.09.026

[4] Iglseder, H., Arens-Fischer, W., and Wolfsberger, W., "Small Satellite Constellations for Disaster Detection and Monitoring," Advances in Space Research, Vol. 15, No. 11, 1995, pp. 79-85. doi:10.1016/0273-1177(95)00077-R

[5] Draim, J. E., "Three-and Four-Satellite Continuous-Coverage Constellations," Journal of Guidance, Control, and Dynamics, Vol. 8, No. 6, 1985, pp. 725-730. doi: $10.2514 / 3.20047$

[6] Walker, J. G., "Circular Orbit Patterns Providing Continuous Whole Earth Coverage," Royal Aircraft Establishment TR 70211, Farnborough, England, U.K., 1970.

[7] Walker, J. G., "Continuous Whole-Earth Coverage by Circular-Orbit Satellite Patterns," Royal Aircraft Establishment TR 77044, Farnborough, England, U.K., 1977.

[8] Denis, G., de Boissezon, H., Hosford, S., Pasco, X., Montfort, B., and Ranera, F., "The Evolution of Earth Observation Satellites in Europe and its Impact on the Performance of Emergency Response Services," Acta Astronautica, Vol. 127, Oct.-Nov. 2016, pp. 619-633. doi:10.1016/j.actaastro.2016.06.012

[9] Voigt, S., et al., "Global Trends in Satellite-Based Emergency Mapping," Science, Vol. 353, No. 6296, 2016, pp. 247-252. doi:10.1126/science.aad8728

[10] Krueger, J., Selva, D., Smith, M., and Keesee, J., "Spacecraft and Constellation Design for a Continuous Responsive Imaging System in Space," AIAA SPACE 2009 Conference \& Exposition, AIAA Paper 2009-6773, 2009. doi: $10.2514 / 6.2009-6773$
[11] Woollands, R. M., Bani Younes, A., and Junkins, J. L., "New Solutions for the Perturbed Lambert Problem Using Regularization and Picard Iteration," Journal of Guidance, Control, and Dynamics, Vol. 38, No. 9, 2015, pp. 1548-1562. doi:10.2514/1.G001028

[12] Wagner, S., Wie, B., and Kaplinger, B., "Computational Solutions to Lambert's Problem on Modern Graphics Processing Units," Journal of Guidance, Control, and Dynamics, Vol. 38, No. 7, 2015, pp. 1305-1311. doi:10.2514/1.G000840

[13] Wang, X., Yue, X., Dai, H., and Atluri, S. N., "Feedback-Accelerated Picard Iteration for Orbit Propagation and Lambert's Problem," Journal of Guidance, Control, and Dynamics, Vol. 40, No. 10, 2017, pp. 2442-2451. doi:10.2514/1.G002638

[14] Bombardelli, C., Gonzalo, J. L., and Roa, J., "Approximate Analytical Solution of the Multiple Revolution Lambert's Targeting Problem," Journal of Guidance, Control, and Dynamics, Vol. 41, No. 3, 2018, pp. 792-801.

doi:10.2514/1.G002887

[15] Guelman, M., and Kogan, A., "Electric Propulsion for Remote Sensing from Low Orbits," Journal of Guidance, Control, and Dynamics, Vol. 22, No. 2, 1999, pp. 313-321. doi: $10.2514 / 2.4380$

[16] Jean, I., and de Lafontaine, I. J., "Autonomous Guidance and Control of an Earth Observation Satellite Using Low Thrust," Advances in the Astronautical Sciences, Vol. 116, No. 3, 2003, pp. 1829-1844.

[17] Zhu, K.-J., Li, J.-F., and Baoyin, H.-X., "Satellite Scheduling Considering Maximum Observation Coverage Time and Minimum Orbital Transfer Fuel Cost," Acta Astronautica, Vol. 66, No. 1, 2010, pp. 220-229. doi:10.1016/j.actaastro.2009.05.029

[18] Davis, J. J., "Constellation Reconfiguration: Tools and Analysis," Ph.D. Thesis, Texas A\&M Univ, College Station, TX, 2010.

[19] De Weck, O. L., Scialom, U., and Siddiqi, A., "Optimal Reconfiguration of Satellite Constellations with the Auction Algorithm," Acta Astronautica, Vol. 62, No. 2, 2008, pp. 112-130. doi:10.1016/j.actaastro.2007.02.008

[20] Legge, R. S., Jr., "Optimization and Valuation of Reconfigurable Satellite Constellations Under Uncertainty," Ph.D. Thesis, Massachusetts Inst. of Technology, Cambridge, MA, 2014.

[21] Paek, S. W., De Weck, O. L., and Smith, M. W., "Concurrent Design Optimisation of Earth Observation Satellites and Reconfigurable Constellations," Journal of the British Interplanetary Society, Vol. 70, No. 1, Jan. 2017, pp. 19-35.

[22] Zhang, G., Cao, X., and Mortari, D., "Analytical Approximate Solutions to Ground Track Adjustment for Responsive Space," IEEE Transactions on Aerospace and Electronic Systems, Vol. 52, No. 3, 2016, pp. 13661383. doi:10.1109/TAES.2016.140644

[23] Zhang, G., and Sheng, J., "Impulsive Ground-Track Adjustment for Assigned Final Orbit," Journal of Spacecraft and Rockets, Vol. 53, No. 4, 2016, pp. 599-609. doi: $10.2514 / 1 . \mathrm{A} 33447$

[24] Co, T. C., "Operationally Responsive Spacecraft Using Electric Propulsion," Ph.D. Thesis, Air Force Inst. of Technology, WrightPatterson AFB, OH, 2012.

[25] Co, T. C., Zagaris, C., and Black, J. T., "Responsive Satellites Through Ground Track Manipulation Using Existing Technology," Journal of Spacecraft and Rockets, Vol. 50, No. 1, 2013, pp. 206-216. doi:10.2514/1.A32263

[26] Co, T. C., and Black, J. T., "Responsiveness in Low Orbits Using Electric Propulsion," Journal of Spacecraft and Rockets, Vol. 51, No. 3, 2014, pp. 938-945. doi:10.2514/1.A32405

[27] Kozai, Y., "The Motion of a Close Earth Satellite," Astronomical Journal, Vol. 64, No. 1274, Nov. 1959, pp. 367-377. doi: $10.1086 / 107957$

[28] Brouwer, D., "Solution of the Problem of Artificial Satellite Theory Without Drag," Astronomical Journal, Vol. 64, No. 1274, Nov. 1959, pp. 378-396. doi: $10.1086 / 107958$

[29] Cerf, M., "Low-Thrust Transfer Between Circular Orbits Using Natural Precession," Journal of Guidance, Control, and Dynamics, Vol. 39, No. 10, 2016, pp. 2232-2239. doi:10.2514/1.G001331

[30] Schaub, H., Vadali, S. R., Junkins, J. L., and Alfriend, K. T., "Spacecraft Formation Flying Control Using Mean Orbit Elements," Journal of the Astronautical Sciences, Vol. 48, No. 1, 2000, pp. 69-87.

[31] Escobal, P. R., Methods of Orbit Determination, Wiley, New York, 1965, pp. 363-371. 
[32] Vallado, D. A., and McClain, W. D., Fundamentals of Astrodynamics and Applications, 3rd ed., Microcosm Press and Springer, Hawthorn, CA, New York, 2007, pp. 615-633, 642-667.

[33] Bate, R. R., Mueller, D. D., and White, J. E., Fundamentals of Astrodynamics, Dover, New York, 1971, pp. 396-407.

[34] Vallado, D. A., and McClain, W. D., Fundamentals of Astrodynamics and Applications, 3rd ed., Microcosm Press and Springer, Hawthorn, CA, New York, 2007, pp. 373-389.

[35] Kidder, S. Q., and Haar, T. H. V., Satellite Meteorology: An Introduction, Gulf Professional Publishing, New York, 1995, pp. 30-41.

[36] Osborne, P., "The Mercator Projections," Zenodo, Edinburgh, 2013, pp. $88-90$. doi:10.5281/zenodo. 35392

[37] Decker, L. B., "World Geodetic System 1984," Defense Mapping Agency, Aerospace Centre TR AD-A167 570, St. Louis, MO, 1986.

[38] Smart, W. M., Text-Book on Spherical Astronomy, 5th ed., Cambridge Univ. Press, New York, 1962, pp. 1-24.

[39] Vallado, D. A., and McClain, W. D., Fundamentals of Astrodynamics and Applications, 3rd ed., Microcosm Press and Springer, Hawthorn, CA, New York, 2007, pp. 549-571.

[40] Committee on Space Research, "CIRA 1972: COSPAR International Reference Atmosphere 1972," Akademie-Verlag, Berlin, 1972.

[41] Hutputtanasin, A., and Tooria, A., "CubeSat Design Specification," California Polytechnic State Univ., Technical Specification, San Luis Obispo, CA, 2005, Revision 9.

[42] Rankin, D., Kekez, D. D., Zee, R. E., Pranajaya, F. M., Foisy, D. G., and Beattie, A. M., "The CanX-2 Nanosatellite: Expanding the Science Abilities of Nanosatellites," Acta Astronautica, Vol. 57, Nos. 2-8, 2005, pp. 167-174 doi:10.1016/j.actaastro.2005.03.032

[43] Krejci, D., Mier-Hicks, F., Fucetola, C., Lozano, P., Schouten, A. H., and Martel, F., "Design and Characterization of a Scalable Ion Electrospray Propulsion System," 30th International Symposium on Space Technology and Science, 34th International Electric Propulsion Conference and 6th Nano-Satellite Symposium, International Symposium on Space Technology and Science (ISTS) Organizing Committee and the Japan Soc. for Aeronautical and Space Sciences (JSASS) Paper IEPC-2015-149/ISTS-2015-b-149, 2015.

[44] Krejci, D., Mier-Hicks, F., Thomas, R., Haag, T., and Lozano, P., "Emission Characteristics of Passively Fed Electrospray Microthrusters with Propellant Reservoirs," Journal of Spacecraft and Rockets, Vol. 54, No. 2, 2017, pp. 447-458. doi:10.2514/1.A33531

[45] Mier-Hicks, F., and Lozano, P. C., "Electrospray Thrusters as Precise Attitude Control Actuators for Small Satellites," Journal of Guidance,
Control, and Dynamics, Vol. 40, No. 3, 2017, pp. 642-649. doi:10.2514/1.G000736

[46] Lozano, P. C., "Less in Space," American Scientist, Vol. 104, No. 5 , 2016, pp. 270-273. doi:10.1511/2016.122.270

[47] Lozano, P. C., Wardle, B. L., Moloney, P., and Rawal, S., "Nanoengineered Thrusters for the Next Giant Leap in Space Exploration," Materials Research Society Bulletin, Vol. 40, No. 10, 2015, pp. 842-849.

doi: $10.1557 / \mathrm{mrs} .2015 .226$

[48] Walker, M., Ireland, B., and Owens, J., "A Set Modified Equinoctial Orbit Elements," Celestial Mechanics, Vol. 36, No. 4, 1985, pp. 409419. doi:10.1007/BF01227493

[49] Dormand, J. R., and Prince, P. J., "A Family of Embedded Runge-Kutta Formulae," Journal of Computational and Applied Mathematics, Vol. 6 , No. 1, 1980, pp. 19-26. doi:10.1016/0771-050X(80)90013-3

[50] "U.S. Standard Atmosphere, 1976," U.S. Committee on Extension to the Standard Atmosphere, National Oceanic and Atmospheric Administration, 1976.

[51] Bogosian, J. R., "Image Collection Optimization in the Design and Operation of Lightweight, Low Areal-Density Space Telescopes," Ph.D. Thesis, Massachusetts Inst. of Technology, Cambridge, MA, 2008.

[52] Paek, S. W., "Reconfigurable Satellite Constellations for Geo-Spatially Adaptive Earth Observation Missions," Ph.D. Thesis, Massachusetts Inst. of Technology, Cambridge, MA, 2012.

[53] Mortari, D., and Wilkins, M. P., "Flower Constellation Set Theory. Part I: Compatibility and Phasing," IEEE Transactions on Aerospace and Electronic Systems, Vol. 44, No. 3, 2008, pp. 953-962. doi:10.1109/TAES.2008.4655355

[54] Castelvi, J., Lancheros, E., Camps, A., and Park, H., "Feasibility of Nano-Satellites Constellations for AIS Decoding and Fire Detection," 4th International Federated and Fractionated Satellite Systems Workshop, International Federated and Fractionated Satellite Systems Workshop Organizing Committee, 2016.

[55] Ross, A. M., Hastings, D. E., Warmkessel, J. M., and Diller, N. P., "Multi-Attribute Tradespace Exploration as Front End for Effective Space System Design," Journal of Spacecraft and Rockets, Vol. 41, No. 1,2004 , pp. $20-28$ doi:10.2514/1.9204

[56] Lowe, C. J., "Methodologies for the Analysis of Value from DelayTolerant Inter-Satellite Networking," Ph.D. Thesis, Univ. of Strathclyde, Glasgow, Scotland, U.K., 2017. 